\title{
Selective G protein signaling driven by Substance P-Neurokinin Receptor structural dynamics
}

Julian A. Harris ${ }^{1,2 *}$, Bryan Faust ${ }^{1,3,4 *}$, Arisbel B. Gondin ${ }^{5,6 *}$, Marc André Dämgen ${ }^{7,8,9,10 *}$, Carl-Mikael Suomivuori ${ }^{7,8,9,10}$, Nicholas A. Veldhuis ${ }^{5,6}$, Yifan Cheng ${ }^{3,11}$, Ron O. Dror ${ }^{7,8,9,10}$, David M. Thal ${ }^{5}$, Aashish Manglik ${ }^{1,12}$

Affiliations:

1. Department of Pharmaceutical Chemistry, University of California, San Francisco, CA, USA

2. Chemistry and Chemical Biology Graduate Program, University of California, San Francisco, CA, USA

3. Department of Biochemistry \& Biophysics, University of California, San Francisco, CA, USA

4. Biophysics Graduate Program, University of California, San Francisco, CA, USA

5. Drug Discovery Biology, Monash Institute of Pharmaceutical Sciences, Monash University, Parkville, Victoria, Australia

6. Australian Research Council Centre of Excellence in Convergent Bio-Nano Science and Technology, Monash University, Parkville, Victoria, Australia

7. Department of Computer Science, Stanford University, CA, USA

8. Department of Molecular and Cellular Physiology, Stanford University School of Medicine, CA, USA

9. Department of Structural Biology, Stanford University School of Medicine, CA, USA 10. Institute for Computational and Mathematical Engineering, Stanford University, CA, USA

11. Howard Hughes Medical Institute, University of California, San Francisco, CA, USA

12. Department of Anesthesia and Perioperative Care, University of California, San Francisco, CA, USA

*These authors contributed equally: Julian A. Harris, Bryan Faust, Arisbel B. Gondin, Marc André Dämgen Correspondence to Yifan Cheng (Yifan.Cheng@ucsf.edu), Ron O. Dror (ron.dror@stanford.edu), David M.Thal (david.thal@monash.edu) or Aashish Manglik (Aashish.Manglik@ucsf.edu) 


\author{
Abstract \\ The neuropeptide Substance P (SP) is important in pain and inflammation. SP activates the \\ neurokinin-1 receptor (NK1R) to signal via $G_{q}$ and $G_{s}$ proteins. Neurokinin $A$ also activates \\ NK1R, but leads to selective $G_{q}$ signaling. How two stimuli yield distinct $G$-protein signaling at \\ the same G-protein-coupled-receptor remains unclear. We determined cryo-EM structures of \\ active NK1R bound to SP or the $\mathrm{G}_{\mathrm{q}}$-biased peptide SP6-11. Peptide interactions deep within \\ NK1R are critical for receptor activation. Conversely, interactions between SP and NK1R \\ extracellular loops are required for potent $G_{s}$ signaling but not $G_{q}$ signaling. Molecular dynamics \\ simulations showed that these superficial contacts restrict SP flexibility deep in the NK1R \\ pocket. SP6-11, which lacks these interactions, is dynamic while bound to NK1R. Structural \\ dynamics of NK1R agonists therefore depend on interactions with the receptor extracellular \\ loops and regulate G-protein signaling selectivity. Similar interactions between other \\ neuropeptides and their cognate receptors may tune intracellular signaling.
}




\section{Introduction}

Substance $\mathrm{P}(\mathrm{SP})$ is a peptide with incredibly diverse roles in animal physiology. Like other neuropeptides, SP exerts long-lasting regulation of synaptic neurotransmission by activating its cognate $\mathrm{G}$ protein-coupled receptor (GPCR), the neurokinin 1 receptor (NK1R). SP action in the nervous system is important in pain, mood, respiration, and nausea ${ }^{1-3}$. Action of SP in other tissues is associated with inflammation or smooth muscle contraction ${ }^{1,2}$. Extensive studies suggest that inhibition of SP activity by NK1R antagonists might lead to effective treatments for pain, inflammation, and mood disorders ${ }^{4,5}$, although the only clinical success to date has been for treatment of chemotherapy-induced nausea and vomiting ${ }^{6,7}$

The NK1R is endogenously activated by SP and another neuropeptide, neurokinin A (NKA). Both SP and NKA belong to the larger family of tachykinin neuropeptides that share a common C-terminal ' $\mathrm{F}(\mathrm{V} / \mathrm{F}) \mathrm{GLM}-\mathrm{NH}_{2}$ ' consensus sequence, which is required for their activity at any of the three neurokinin receptors. The more divergent $\mathrm{N}$-terminal region of tachykinin peptides has previously been implicated in dictating which neurokinin receptor a tachykinin prefers ${ }^{8,9}$. Like other neuropeptides, tachykinin function follows the "message-address" framework, in which two distinct portions of a peptide encode either the efficacy (message) or receptor selectivity (address) $^{10-12}$. Following this framework, NKA was initially described as specific for the neurokinin 2 receptor $(\mathrm{NK} 2 \mathrm{R})^{8,13}$. However, both SP and NKA activate NK1R in cell lines and in various physiological settings ${ }^{14-18}$.

Intriguingly, activation of NK1R by SP or NKA induces distinct cellular responses and, in certain tissues, distinct physiological outcomes ${ }^{16,19-21}$. SP increases both inositol phosphate (IP) and cAMP second messengers downstream of $G_{q}$ and $G_{s}$ signaling pathways, respectively ${ }^{17,18,22}$. By contrast, NKA signals potently via $G_{q}$ but has decreased $G_{s}$ stimulatory activity ${ }^{17,18}$. Molecular pharmacology studies revealed that SP binding to NK1R is distinct from NKA binding ${ }^{18,23,24}$. A common model proposed by these studies is that NK1R exists in two distinct active conformations: an SP-selective state and a general-tachykinin state that binds both SP and $\mathrm{NKA}^{18,24}$. Mutations can alter the relative proportion of these two states, yielding changes in the measured affinities for SP, NKA and related tachykinins ${ }^{18,24}$. Intriguingly, these mutations also dramatically affect the ability of NK1R to signal via $G_{q}$ or $G_{s}$, suggesting that these distinct active conformations are coupled to distinct signaling outcomes ${ }^{18}$. 
The ability of two agonists to induce distinct intracellular signaling cascades downstream of a single GPCR is well established. However, how two endogenous stimuli yield distinct G protein coupling preference at the same receptor remains unclear at the biochemical and structural level. Here, we combine structural biology with molecular dynamics simulations and cellular signaling studies to decipher the molecular basis of agonist-dependent $\mathrm{G}$ protein-selective signaling at the NK1R.

\section{Structure of the Substance P-NK1R-miniG ${ }_{\mathrm{s} / \mathrm{q} 70}$ complex}

To enable structure determination of active human NK1R without thermostabilizing mutations or truncations, we generated a construct with the engineered $G$ a subunit miniG $\mathrm{s}_{\mathrm{s} 70}$ fused to the C-terminus of the receptor ${ }^{25}$. The miniG $_{\mathrm{s} / \mathrm{q} 70}$ protein presents the GPCR-interacting a5 helix of $\mathrm{Ga}_{\mathrm{q}}$ on an engineered $\mathrm{Ga}_{\mathrm{s}}$ protein stabilized in the active conformation and with complete truncation of the Ga alpha-helical domain. This strategy improved the biochemical stability of Substance P (SP)-bound receptor compared to full-length NK1R alone (Supplementary Fig. 1). Purified SP-bound NK1R-miniG ${ }_{s / q 70}$ fusion protein was mixed with excess G $\beta y$ and nanobody 35 $(\mathrm{Nb} 35)^{26}$ for structure determination by cryogenic-electron microscopy (cryo-EM) (Supplementary Fig. 1).

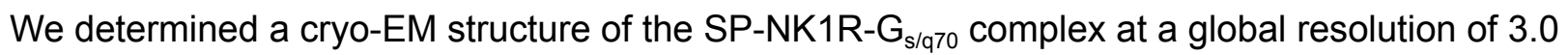
$\AA$ (Fig. 1A and Supplementary Fig. 2). As is common for many GPCR-G protein complex structures, our initial maps yielded poor resolution for SP, the orthosteric binding pocket, and the extracellular loops. To improve reconstruction in these regions, we performed iterative focused refinements using a mask encompassing only the upper transmembrane region of the 7TM bundle. The resulting improved maps enabled an atomic model for all subunits of the complex and the SP peptide (Fig. 1B, Supplementary Fig. 2, Supplementary Fig. 3).

SP-activated NK1R is in a distinct active conformation when compared to other class A GPCRs. Like other GPCRs, active NK1R displays an $8.3 \AA$ movement of transmembrane helix 6 (TM6) away from the 7TM helical bundle, enabling insertion of the C-terminal $\alpha 5$-helix of the miniG $_{s / q 70}$ protein (Fig. 1C). This movement is associated with other conserved changes in class A GPCR activation, including displacement of the $\mathrm{W}^{6.48}$ 'toggle-switch' (superscripts denote Ballesteros-Weinstein numbering ${ }^{27}$ ), rearrangement of the ' $\mathrm{P}^{5.50} \mathrm{I}^{3.40} \mathrm{~F}^{6.44}$ ' connector motif, and movement of the ' $D^{3.49} R^{3.50} Y^{3.51}$ ' motif (Supplementary Fig. 4) ${ }^{28}$. These conformational changes 
link ligand binding in the orthosteric site to the intracellular $G$ protein coupling site and facilitate G protein binding.

By contrast, the conserved ' $N^{7.49} P^{7.50} x x Y^{7.53 '}$ motif in the SP-NK1R-miniG ${ }_{s / 970}$ structure remains in an inactive conformation. A hallmark of class A GPCR activation is inward movement of TM7 into the helical core ${ }^{28}$ (Fig. 1D). This allows $Y^{7.53}$ of the NPxxY motif to engage in an extended water-mediated hydrogen bonding-network with other residues on the cytoplasmic face of TM3 and TM5, as observed for the active $\mu$ opioid receptor ${ }^{29}$ (Fig. 1E). This inward movement of $Y^{7.53}$ is not observed in the SP-NK1R-miniG $\mathrm{s}_{\mathrm{s} / 0}$ structure and TM7 remains in a conformation that closely resembles the inactive-state (Fig. 1E). Importantly, other structures of GPCRs solved in complex with $\mathrm{G}_{\mathrm{q} / 11}$ family $\mathrm{G}$ proteins, $\operatorname{mini}_{\mathrm{s}}$, and $\operatorname{mini}_{\mathrm{s} / \mathrm{q} 70}$ proteins show canonical inward movement of TM7 upon receptor activation ${ }^{30-34}$.

The unique active-state of SP-bound NK1R resembles a previously determined structure of the neurotensin 1 receptor $\left(N T S_{1} R\right)$ bound to the cAMP inhibitory $G$ protein $G_{i}^{35,36}$. Two active-state conformations of $N_{T S} R$ bound to $G_{i}$ have previously been observed: a canonical state with inward movement of TM7 and a 'non-canonical' state without TM7 rearrangement ${ }^{35}$ (Fig. 1E). Although the seven transmembrane domain of $\mathrm{NK} \mathrm{R}$ bound to $\operatorname{miniG}_{\mathrm{s} / \mathrm{q} 70}$ is in a similar conformation to the 'non-canonical' NTS ${ }_{1}$ R conformation, we do not observe the $45^{\circ}$ rotation of the $G$ protein observed for 'non-canonical' NTS ${ }_{1} R$ (Supplementary Fig. 4). While there are important caveats to our interpretation of the interactions between NK1R and the engineered miniG $_{s / q 70}$ protein, we surmise that fully active NK1R bound to the C-terminus of $G_{q}$ exists in a unique conformation compared to most class A GPCRs.

Unlike the majority (98\%) of class A GPCRs, NK1R possesses a glutamate residue at the highly conserved $D^{2.50}$ position ${ }^{37,38}$. In inactive-state class A GPCRs, this canonical $D^{2.50}$ residue participates in an extended, water-mediated hydrogen-bonding network between TM helices 2,3,6 and 7. For most GPCRs, activation is coupled with an inward movement of TM7 driven by a direct interaction between $D^{2.50}$ and $N^{7.49}$ (Supplementary Fig. 4) of the NPxxY motif. By contrast, $\mathrm{E} 78^{2.50}$ forms a direct interaction with $\mathrm{N} 301^{7.49}$ in the NK1R inactive-state. We speculate that the stable and direct $E^{2.50}-\mathrm{N}^{7.49}$ interaction in the NK1R inactive-state disfavors inward TM7 motion during activation and contributes to the 'non-canonical' active-state. Indeed, prior work has shown that disrupting the $\mathrm{E}^{2.50}-\mathrm{N}^{7.49}$ interaction with mutagenesis selectively 
diminishes $G_{s}$ signaling but does not affect $G_{q}$ signaling ${ }^{39}$, suggesting that the 'non-canonical' NK1R active conformation is important for robust $G_{s}$ and $G_{q}$ signaling downstream of NK1R activation.

\section{Molecular Recognition of Substance P by NK1R}

SP binds with an expansive interface stretching from a deeply-buried 7TM pocket to the distal portions of the NK1R ECL2 and N-terminus (Fig. 2A). We observed clearly resolved cryo-EM density for SP C-terminal residues 6-11, enabling us to unambiguously model this portion of the peptide (Fig. 2A). The $\mathrm{N}$-terminal portion of SP, including residues 1-5, interact primarily with the extracellular loop 2 (ECL2) and the N-terminus of NK1R. The density for these residues is less well resolved, but we were able to confidently place all mainchain atoms and all side chains with the exception of R1 and K3.

SP binds to NK1R in a distinct manner compared to other neuropeptides at their cognate receptors. We compared the binding of SP to the $N_{T S} R$ bound to neurotensin $8-13^{40}$, the $\mu$-opioid receptor bound to the peptide mimetic agonist DAMGO ${ }^{41}$, and the orexin 2 receptor bound to orexin $\mathrm{B}^{42}$ (Supplementary Fig. 5). All of these neuropeptides make extensive contacts with the deep 7TM pocket, likely important for determining their efficacy as agonists for their respective receptors. The extended conformations of the peptides in the receptor binding pockets enable further interactions with the extracellular loops. In contrast to the binding of these other neuropeptides at their cognate receptors, SP makes more extensive contacts with ECL2 and the $\mathrm{N}$-terminus of NK1R, manifesting as an outward displacement of the extracellular tip of TM1 and a more ordered $\mathrm{N}$ terminus (Supplementary Fig. 5).

The SP orthosteric binding pocket is distinct from the binding sites of NK1R antagonists determined in previous inactive-state structures ${ }^{38,43,44}$. For example, the antagonist netupitant ${ }^{38}$ (PDB: 6HLP) minimally overlaps with SP (Fig. 2B), with only the 2-methylphenyl and 3,5-bis(trifluoromethyl)phenyl groups of netupitant binding in the same region as M11 of SP (Fig. 2B). The core of the netupitant antagonist scaffold, however, extends along TM4 and TM5 toward the extracellular region of the receptor in a portion of the orthosteric pocket that is not occupied by SP. All structurally characterized NK1R antagonists possess a similar molecular scaffold to netupitant and bind to a relatively small portion of the total SP binding site (Supplementary Fig. 5). This distinct binding topology is consistent with prior mutagenesis data, which found only two 
NK1R residues, Q165 $5^{4.60}$ and $\mathrm{Y} 287^{7.35}$, are important for both SP and non-peptide antagonists binding $^{9,45-49}$ (Supplementary Fig. 5).

The expansive SP-NK1R interface is consistent with prior mutagenesis efforts, which found that residues both within the deep 7TM site and the NK1R N-terminus potently reduce SP binding affinity $^{9,47,49}$. Our structure of the SP-NK1R complex revealed that the amidated C-terminus of SP forms an extensive hydrogen-bonding network with NK1R residues N85 ${ }^{2.57}$, N89 ${ }^{2.61}, \mathrm{H} 108^{3.28}$, and Y287 7.35 (Fig. 2D). To finely probe the importance of specific hydrogen bonds in SP binding to NK1R, we tested the ability of SP to activate NK1R mutants with conservative amino acid substitutions at these key positions in a $\mathrm{Ca}^{2+}$ mobilization assay. In contrast to the dramatic loss of potency previously observed with non-conservative alanine mutations at these sites $^{49}$, we observed relatively minor changes in SP potency or maximal efficacy with these conservative mutations (Fig. 2E and Supplementary Fig. 6).

We hypothesized that other SP-NK1R interactions, perhaps those in the extracellular regions of the receptor, could compensate for the disrupted hydrogen bonding network in the deep portion of the NK1R pocket. To test this, we examined the potency and efficacy of a truncated version of SP containing only residues 6-11 (SP6-11, Fig. 3A), which would be unable to interact with the NK1R ECL2 and N-terminus. As observed previously ${ }^{18}$, we found that SP6-11 is equally potent as SP in stimulating $\mathrm{Ca}^{2+}$ signaling and IP1 accumulation at wild-type NK1R (Fig. 3B and Supplementary Fig. 6). When tested against our conservative NK1R mutants targeting the SP C-terminal amide hydrogen bonding network, we observed a dramatic 30-1000 fold loss in potency for SP6-11 (Fig. 2E). We therefore conclude that the extensive hydrogen bonding network recognizing the amidated C-terminus of SP is indeed important for $\mathrm{Ca}^{2+}$ signaling, but that the extended contacts between SP and NK1R in the extracellular regions serve an important role in NK1R signaling beyond simply dictating the tachykinin receptor subtype selectivity of SP.

\section{Structural interrogation of NK1R signaling bias}

While SP potently activates both $G_{q}$ and $G_{s}$ signaling downstream of NK1R ${ }^{22}$, NKA and $\mathrm{N}$-terminally truncated $\mathrm{SP}$ analogs are weaker agonists of $\mathrm{G}_{\mathrm{s}}$ signaling ${ }^{17,18}$. We confirmed these prior results in signaling studies. SP, NKA, and SP6-11 produced equally potent and efficacious $\mathrm{Ca}^{2+}$ and IP signaling responses (Fig. 3B and Supplementary Fig. 6). By contrast, NKA and 
SP6-11 were 6- and 16-fold less potent than SP in eliciting cAMP accumulation, respectively (Fig. 3C, Supplementary Table 2), confirming their $\mathrm{G}_{\mathrm{q}}$ selective signaling profiles.

Prior pharmacology studies have demonstrated that SP and its analogs bind to NK1R in two distinct conformations that likely depend on the specific $\mathrm{G}$ protein coupled to the receptor ${ }^{24,50}$. Indeed, NK1R-G and NK1R-G fusion proteins display different binding affinities for $\mathrm{SP}^{50}$, suggesting that $\mathrm{G}_{\mathrm{q}}$ - and $\mathrm{G}_{\mathrm{s}}$-coupled NK1R exist in distinct conformations. We therefore reasoned that additional cryo-EM structures of active NK1R may provide insight into how SP and other tachykinins induce distinct $\mathrm{G}_{\mathrm{q}}$ and $\mathrm{G}_{\mathrm{s}}$ signaling outcomes. In particular, we speculated that differences in the SP-ECL2 interaction interface may explain the diminished $\mathrm{G}_{\mathrm{s}}$ agonism of NKA and SP6-11. To explore how SP induces $\mathrm{G}_{\mathrm{s}}$ signaling, we determined a structure of SP-NK1R bound to a miniG protein analog of $\mathrm{G}_{\mathrm{s}}\left(\operatorname{miniG}_{\mathrm{s} 399}\right)$ at $3.1 \AA$ resolution (Fig. 3D, Supplementary Fig. 1, Supplementary Fig. 7). Furthermore, to understand how NKA and SP6-11 induce $\mathrm{G}_{\mathrm{q}}$ selective NK1R signaling, we determined the structure of a SP6-11-NK1R-miniG ${ }_{s / q 70}$ complex at $3.2 \AA$ resolution (Fig. 3E, Supplementary Fig. 1, Supplementary Fig. 8).

The structure of the full-length SP-NK1R-miniGs ${ }_{399}$ complex is almost identical to our SP-NK1R-miniG ${ }_{s / q 70}$ complex, with overall NK1R root mean square deviation (RMSD) of $0.38 \AA$. Importantly, we observe clearly resolved EM density for the N-terminus of SP, which interacts with the NK1R extracellular regions in a very similar manner to the SP-NK1R-miniG $\mathrm{s} / \mathrm{q} 70$ structure (Supplementary Fig. 9). The similarity in these SP-NK1R-miniG protein structures suggests that the overall conformation of $G_{q^{-}}$and $G_{s}$-coupled NK1R may be similar while bound to SP. However, there are important caveats to this interpretation. First, the primary interaction between NK1R and the miniG proteins is the insertion of the $G$ protein C-terminal $\alpha 5$-helix into the receptor 7TM core. In the chimeric miniG $_{\mathrm{s} / \mathrm{q} 70}$ protein, the $\alpha 5$-helix is derived from $\mathrm{G}_{\mathrm{q}}$, whereas the remainder of the miniG $\mathrm{s}_{\mathrm{s} / \mathrm{q} 0}$ protein is derived from $\mathrm{G}_{\mathrm{s}}$. It is possible that the interaction between NK1R and wild-type $G_{q}$ may be different from what we observe with mini $G_{s / q 70}$ here. Furthermore, both miniG proteins used here have been engineered to stabilize the active $G$ protein conformation and increase the affinity of the G protein-GPCR interaction. We cannot rule out the possibility that these modifications may influence the specific conformation of NK1R that we observed in our structures. Finally, our cryoEM reconstructions capture a single, low-energy state of a nucleotide-free NK1R-miniG protein complex. It is also possible that $G_{q}$ and $G_{s}$ signaling 
selectivity arises from transient NK1R G protein-coupled states that are not structurally observed in our work.

The structure of SP6-11 activated NK1R-miniG ${ }_{\text {s/q70 }}$ also revealed a highly similar receptor-G protein conformation when compared to full-length SP, with notable exceptions in the peptide binding site (Fig. 3F,G and Supplementary Fig. 9). We observed a shorter density for SP6-11 in the orthosteric binding pocket, consistent with the N-terminal truncation of SP. The cryo-EM density for SP6-11 is comparatively worse than the density for both full-length SP reconstructions when viewed with unsharpened maps at the same enclosed volume threshold (Fig. 3G).

Specifically, there is a lack of continuous electron density between the peptide backbone and the F7 sidechain and the electron density for the M11 sidechain is completely missing. By contrast, the density for the receptor is comparatively well resolved for both full-length SP and SP6-11 structures, suggesting that the weaker density we observed for SP6-11 does not arise from local resolution artifacts (Supplementary Fig. 9). Selectively weaker density for F7 and M11 may arise from increased dynamic motion of SP6-11 compared to full-length SP. We speculate that increased SP6-11 dynamic motion may result from a lack of stabilizing contacts between the $\mathrm{N}$-terminus of SP and the extracellular regions of NK1R, potentially leading to both increased sensitivity of SP6-11 to mutations in the deep 7TM pocket (Fig. 2E) and decreased potency of SP6-11-mediated $\mathrm{G}_{\mathrm{s}}$-signaling (Fig. 3C).

\section{Truncation of SP N-terminus increases $\mathrm{C}$-terminus mobility}

We turned to all-atom molecular dynamics (MD) simulations to understand the mechanism by which SP N-terminal truncation leads to $G_{q}$ selective signaling. To this end, we performed 12 independent $2-\mu$ s simulations of active NK1R bound to SP and another 12 of active NK1R bound to SP6-11.

In our simulations, SP6-11 is less restrained in its motion than the corresponding C-terminal residues of SP and explores more space within the binding pocket (Fig. 4). SP6-11 residues F7 and M11 exhibit particularly notable differences in dynamics compared to SP. In simulations of NK1R bound to full-length SP, the F7 side chain remains mostly between TM7 and TM2, as in the SP-bound NK1R structure (Fig. 4A). In simulations with SP6-11, on the other hand, the F7 side chain samples a wider range of orientations (Fig. 4C). The side chain of M11 adopts two major orientations in simulations of SP: one pointing between TM5 and TM6 as in the SP-bound 
structure of NK1R and one pointing between TM6 and TM7. For SP6-11, we observe a wider range of M11 side chain orientations with additional conformations pointing towards TM6 and TM7 (Fig. 4C). Altogether, we observe a significant increase in the root mean square fluctuation (RMSF) for SP6-11 bound to NK1R, both for the entire C-terminal peptide region and for the F7 and $\mathrm{M} 11$ residues (Fig. 4B). This increased flexibility is consistent with poorly resolved regions for the SP6-11 peptide in our cryo-EM structure (Fig. 3G). We conclude that disruption of the interactions between the SP N-terminus and the NK1R leads to destabilization of SP C-terminal residues.

Intriguingly, the different orientations observed for M11 and F7 affect contacts with TM7. Given that active NK1R is already in an unusual 'non-canonical' conformation, with TM7 inactive but TM6 in an outward position, it is tempting to speculate that these different interactions with TM7 could also stabilize distinct intracellular conformations differing in TM7 conformation. Different TM7 conformations have been previously shown to mediate differential signaling responses of another peptidergic GPCR, the angiotensin II type 1 receptor ${ }^{51}$.

\section{Disruption of SP contacts with ECL2 biases signaling}

Our simulations suggested that the interactions of the N-terminal region of SP with NK1R ECL2 serve to stably position the C-terminus of the peptide. Disruption of these interactions may therefore destabilize the $\mathrm{C}$-terminal region of $\mathrm{SP}$ and achieve similar $\mathrm{G}_{\mathrm{q}}$ preferential signaling as observed for SP6-11. To directly test the relevance of interactions between ECL2 and SP, we designed NK1R mutations that disrupt the SP-ECL2 interface (Supplementary Fig. 10). Two such mutations, $\mathrm{M} 174 \mathrm{I}$ and $\mathrm{R} 177 \mathrm{M}$, displayed $\mathrm{G}_{\mathrm{q}}$ preferential signaling by SP. In structures of NK1R bound to SP, M174 makes hydrophobic contacts with F8 of SP while R177 forms an extended hydrogen-bond network with the SP backbone and NK1R residues N96 ${ }^{2.68}$ in TM2 and N23 in the $\mathrm{N}$-terminus (Fig. 5A). In signaling studies, both M174I and R177M are equally potent and efficacious as wild-type NK1R at $\mathrm{Ca}^{2+}$ mobilization (Fig. 5B). By contrast, both of these mutations significantly decrease cAMP production by SP, with R177M displaying a 20-fold reduction in potency and a >3-fold reduction in efficacy compared to wild-type NK1R (Fig. 5C, Supplementary Table 2). Importantly, these mutants are expressed at similar levels as wild-type NK1R (Supplementary Fig. 10). Contacts between SP and NK1R ECL2 are therefore critically important for potent $\mathrm{G}_{\mathrm{s}}$-coupled cAMP signaling. 


\section{Conclusion}

Substance $P$ is a prototypical member of the broader family of neuropeptides that act at GPCRs to modulate neuronal function. Our structures of full-length SP bound to active NK1R revealed an extensive contact interface with NK1R, stretching from the deeply buried regions in the 7TM domain to the extracellular regions of the receptor. A network of specific hydrogen bonds between the amidated C-terminus of SP and the deep orthosteric pocket of NK1R are important for peptide recognition; removal of specific hydrogen bonds impairs the ability of SP6-11 to activate NK1R. Our structures also reveal important contacts between the SP N-terminus and the extracellular loops of NK1R, providing insight into how less-conserved sequences in neuropeptides engage GPCRs. While our structural views of full-length neuropeptides bound to their cognate GPCRs remains limited to only a few other examples ${ }^{42,52}$, an emerging theme is that peptides bind in an extended manner, with regions of the peptide engaging the receptor extracellular loops.

Our work revises the 'message-address' model for peptidergic signaling at GPCRs. In particular, we demonstrate that interactions between the N-terminal region of SP and the extracellular loops of NK1R, which were previously characterized as conferring receptor subtype selectivity, are also required for balanced signaling via both the $G_{q}$ and $G_{s}$ signaling pathways. Loss of these interactions, due to either truncation of SP's N-terminus or NK1R ECL2 mutations, leads to $\mathrm{G}_{\mathrm{q}}$-selective signaling. Other endogenous tachykinins that signal selectively via $\mathrm{G}_{\mathrm{q}}$, such as $N K A$, likely do so because they lack sequences that can engage the NK1R extracellular loops. Neuropeptide regions that engage GPCR extracellular loops may therefore specify not only which receptor subtype a peptide preferentially engages but also the signaling outcomes downstream of a specific GPCR.

Our work has broader implications for other neuropeptide GPCRs. Pharmacological studies with other neuropeptides, including opioid peptides ${ }^{53,54}$, neuropeptide $S^{55,56}$, and neuropeptide $Y^{57}$ suggest that interactions between the divergent, less conserved regions of the neuropeptide and their cognate receptor extracellular loops, which also diverge in sequence, can tune signaling efficacy via multiple $\mathrm{G}$ proteins or $\beta$-arrestins. Our work highlights the role of peptide-receptor extracellular contacts in determining the conformational flexibility of the core "message" region of SP. A similar mechanism may be responsible for fine-tuning intracellular signaling, or for promoting a complete signaling response when multiple endogenous neuropeptides are present 
bioRxiv preprint doi: https://doi.org/10.1101/2021.05.16.444192; this version posted May 17, 2021. The copyright holder for this preprint (which

was not certified by peer review) is the author/funder, who has granted bioRxiv a license to display the preprint in perpetuity. It is made available under aCC-BY-NC-ND 4.0 International license.

and acting at a single GPCR. Assessing the structure and dynamics of neuropeptides bound to their cognate receptors thus promises to yield a mechanistic understanding of what drives GPCR signaling complexity, and may eventually provide a path to control such complex signaling with designed molecules. 


\section{FIGURES}
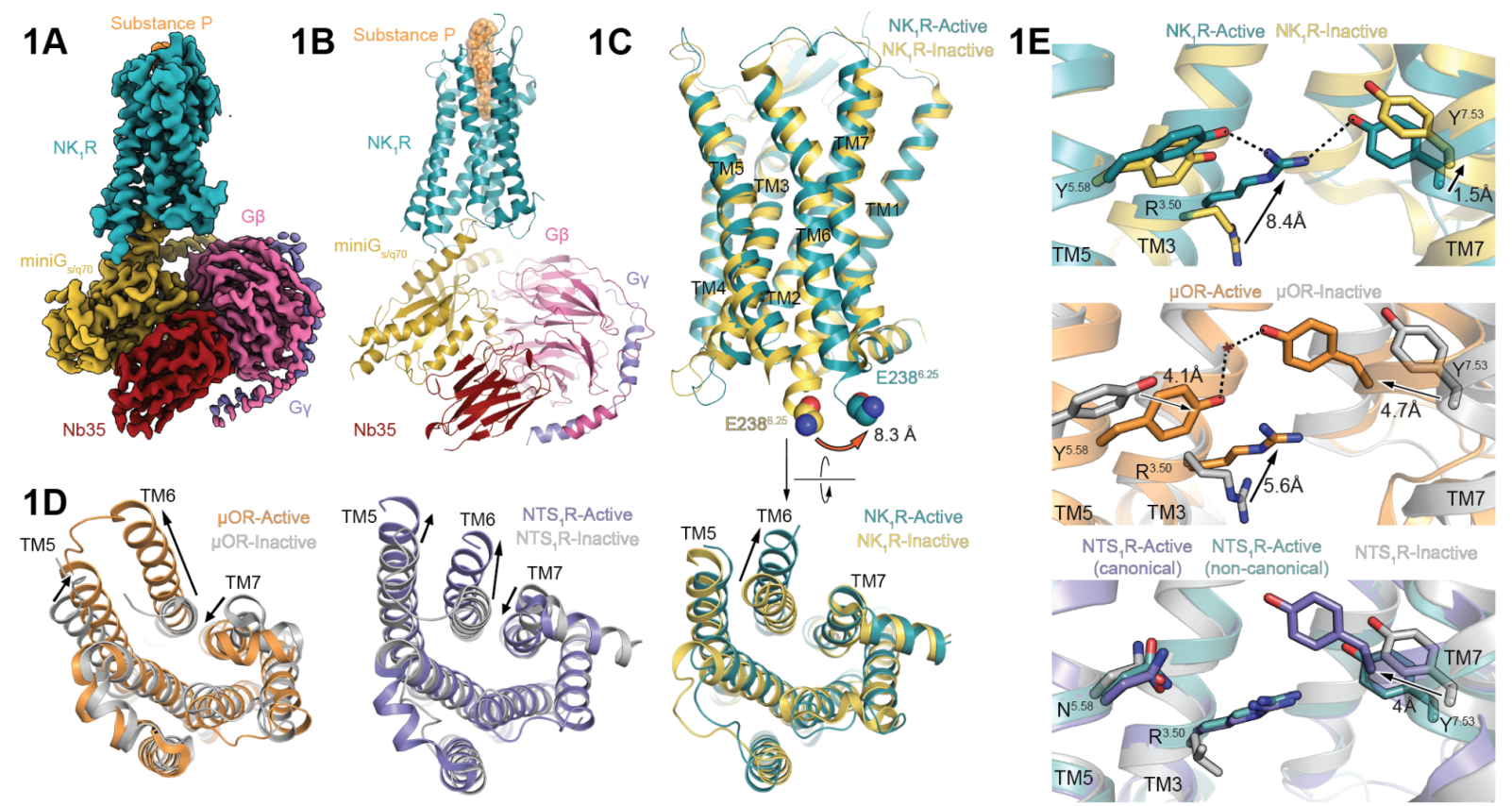

Figure 1. Cryo-EM structure of active NK1R bound to Substance $P$

(A) Unsharpened cryo-EM map of Substance P-bound NK1R-miniG ${ }_{\mathrm{s} / \mathrm{q} 70}-\mathrm{Nb} 35$ complex.

(B) Ribbon diagram of $\mathrm{NK}_{1} \mathrm{R}-\mathrm{mini}_{\mathrm{s} / \mathrm{q} 70}-\mathrm{Nb} 35$ complex. Substance $\mathrm{P}$ is shown as orange spheres.

(C) Alignment of active $\mathrm{NK}_{1} \mathrm{R}$ with inactive, antagonist-bound NK1R (PDB: $6 \mathrm{HLP}^{38}$ ) shows $8.3 \AA$ outward displacement of TM6.

(D) Comparison of active $\mathrm{NK}_{1} \mathrm{R}$ to other active-state GPCRs shows minimal inward movement of TM7 upon activation. Activation-dependent inward movement of TM7 for two class A neuropeptide GPCRs is shown for comparison: $\mu$-opioid ( $\mu \mathrm{OR}$, active PDB: $5 C 1 \mathrm{M}^{29}$, inactive PDB: 4DKL ${ }^{58}$ ) and neurotensin 1 (NTS ${ }_{1}$, active PDB: $6 \mathrm{OS9}^{35}$, inactive PDB: 4BUO ${ }^{59}$ ).

(E) The active-state NK1R NPxxY motif shows a similar conformation to the 'non-canonical' active-state $\mathrm{NTS}_{1} \mathrm{R}$ conformation $\left(\mathrm{PDB}: 6 \mathrm{OSA}^{35}\right.$ ). Inward movement of $\mathrm{Y}^{7.53}$ and TM7 for canonically active $\mu \mathrm{OR}$ and $\mathrm{NTS}_{1} \mathrm{R}$ is shown for comparison. 


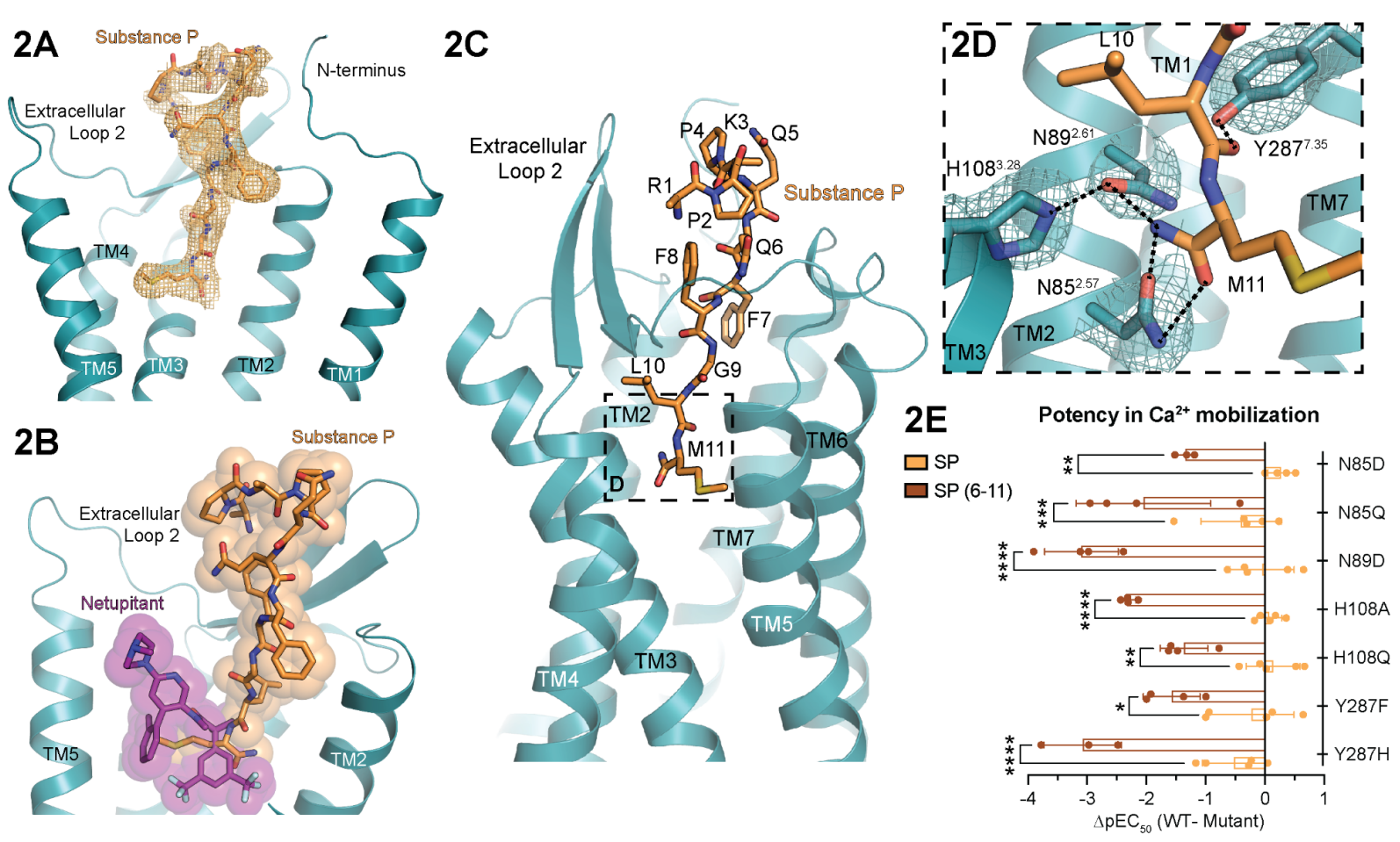

Figure 2. Molecular recognition of Substance $P$ by NK1R

(A) Sharpened cryo-EM density map for Substance P (SP) in the NK1R binding pocket shown as orange mesh and contoured at a distance of $1.85 \AA$ from the placement of Substance $P$ atoms.

(B) Overlay of Substance P and netupitant binding sites in NK1R orthosteric site.

(C) Substance $P$ forms an extensive interaction interface with NK1R, reaching from the deep orthosteric pocket to the distal extracellular regions.

(D) The C-terminally amidated methionine of Substance P (M11) forms an extended hydrogen-bonding network with NK1R. Sharpened cryo-EM density map for sidechains is contoured $1.85 \AA$ away from modeled atoms.

(E) Truncated SP6-11 is sensitive to mutations in the deep orthosteric pocket, highlighting the importance of the extended hydrogen-bond network for Substance $\mathrm{P}$ recognition. Bar graphs represent mean $\triangle p E C_{50}$ (WT - Mutant) \pm s.e.m. from $n \geq 3$ independently fit biological replicates. Statistical significance between SP and SP6-11 $\triangle \mathrm{pEC}_{50}$ for each mutant is compared in a two-way analysis of variance (ANOVA) with Šídák's multiple comparison-corrected post hoc test, $\left({ }^{*}=p \leq 0.033,{ }^{* *}=p \leq 0.002,{ }^{* * *}=p \leq 0.0002,{ }^{* * *}=p \leq 0.0001\right)$. Full quantitative parameters from this experiment are listed in Supplementary Table 3. 
3A

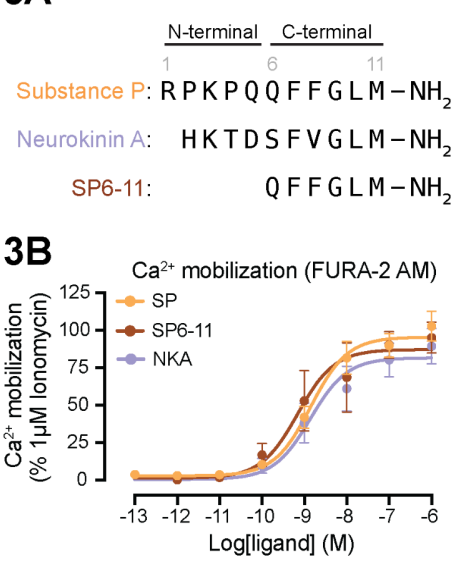

$3 \mathrm{C}$

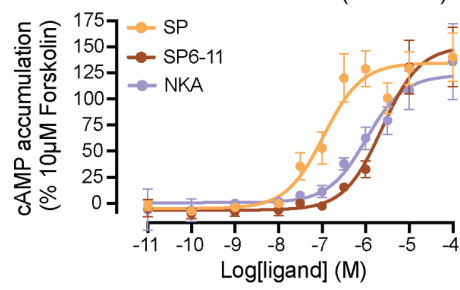

$3 \mathrm{D}$
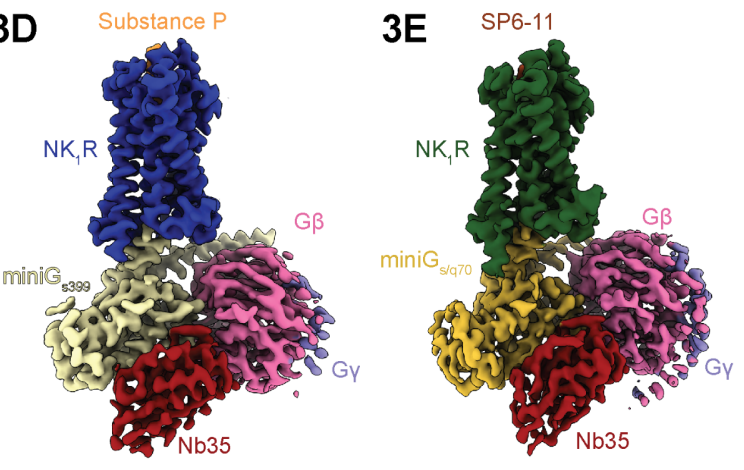

$3 F$

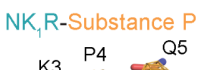

3G $\quad \mathrm{NK}_{1} \mathrm{R}-\mathrm{SPG}-11$

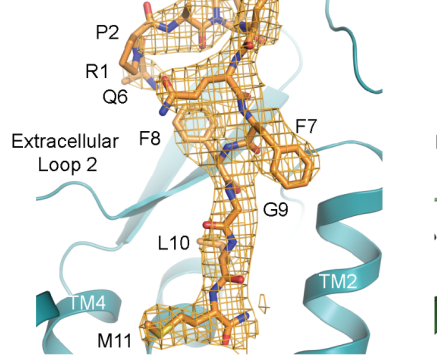

Figure 3. Structural interrogation of SP6-11, a $\mathbf{G}_{\mathrm{q}}$-selective tachykinin

(A) Sequence of SP, Substance P 6-11 (SP6-11) and Neurokinin A (NKA).

$(B, C) \mathrm{Ca}^{2+}$ and cAMP signaling assays demonstrate that SP6-11 and NKA signal potently through $G_{q}$ but have diminished potency for $G_{s}$ signaling. Signaling graphs represent the global fit of grouped data \pm s.e.m. from $n \geq 3$ independent biological replicates. Full quantitative parameters from this experiment are listed in Supplementary Table 2.

(D) Cryo-EM map of SP-NK1R-miniG ${ }_{\mathrm{s} 399}-\mathrm{Nb} 35$ complex.

(E) Cryo-EM map of SP6-11-bound NK1R-miniG $\mathrm{s}_{\mathrm{s} / \mathrm{70}}-\mathrm{Nb} 35$ complex.

$(\mathbf{F}, \mathbf{G})$ Unsharpened density maps at equivalent enclosed volume thresholds for SP and SP6-11 are shown as mesh. For SP6-11, density for the M11 side chain is absent, as is connecting density for the F7 side chain (indicated by red arrows). By contrast, these regions are clearly resolved for SP. Density map for the peptide is contoured $2 \AA$ away from modeled atoms. 


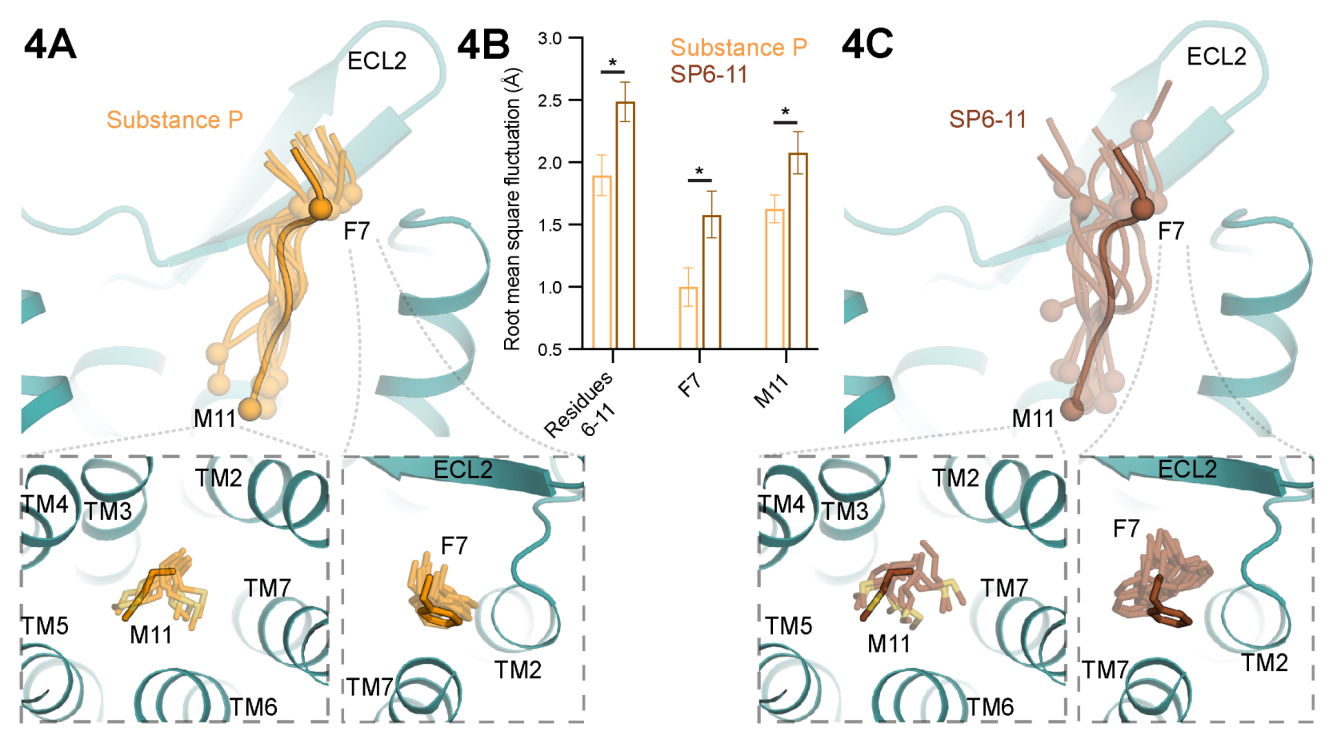

Figure 4. Molecular dynamics shows increased mobility of SP6-11.

(A) Molecular dynamics simulation snapshots for backbone of C-terminal residues of Substance $\mathrm{P}$ (SP). Ca atoms for methionine 11 (M11) and phenylalanine 7 (F7) are shown as spheres. The starting cryo-EM structure of SP in the binding pocket is shown with outline; simulation snapshots are transparent. Simulations included all 11 amino-acids of SP, but only residues 6-11 are shown here to enable comparison with SP6-11. Insets show conformations for F7 and M11 side chains of SP.

(B) Quantitation of peptide mobility in molecular dynamics simulations as measured by root mean square fluctuation (RMSF). Bar graphs show mean RMSF \pm s.e.m. from twelve independent molecular dynamics simulations $\left({ }^{*}=p<0.05\right.$, two-sided Welch's t-test).

(C) Simulation snapshots for SP6-11. Insets show alternative conformations for F7 and M11 side chains of SP6-11. 


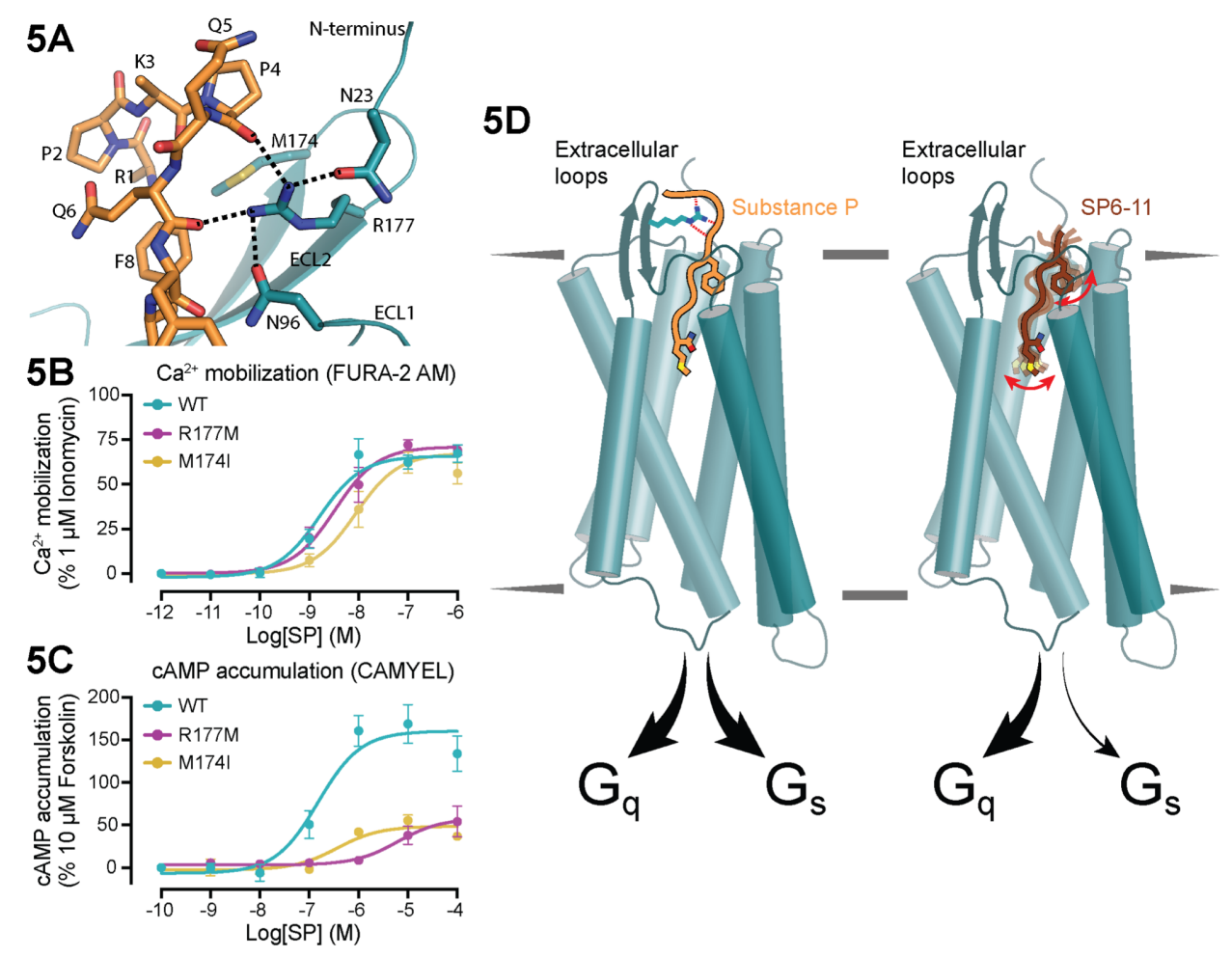

Figure 5. Disruption of SP-NK1R ECL2 contacts leads to $\mathbf{G}_{\mathrm{q}}$-selective signaling (A) NK1R ECL2 contacts with the N-terminal region of SP. R177 engages in an extended hydrogen bonding network with the SP backbone while M174 makes van der Waals contacts with R1 and P4 $(B, C) \mathrm{Ca}^{2+}$ and $\mathrm{CAMP}$ signaling assays for point mutants disrupting ECL2-SP interactions. Disruption of SP-NK1R ECL2 contacts leads to strongly $G_{q}$ selective signaling. Signaling graphs represent the global fit of grouped data \pm s.e.m. from $n \geq 3$ independent biological replicates. Full quantitative parameters from this experiment are listed in Supplementary Table 3.

(C) Model for tuning of G protein selectivity driven by contacts between SP and ECL2. 


\section{METHODS}

\section{Expression and purification of full-length Substance P-NK1R-miniG s/q70 $_{\text {complex }}$}

For structure determination, human TACR1 with an N-terminal HA signal sequence followed by a FLAG epitope tag was cloned into a pcDNA ${ }^{\mathrm{TM}} 3.1 / \mathrm{Zeo}^{(+)}$vector containing a tetracycline-inducible expression cassette. The miniG $_{\mathrm{s} / \mathrm{q} 70}$ protein $^{25}$ was fused to the NK1R C-terminus, preceded by a flexible glycine/serine linker and rhinovirus $3 \mathrm{C}$ protease recognition site (LEVLFQGP). The

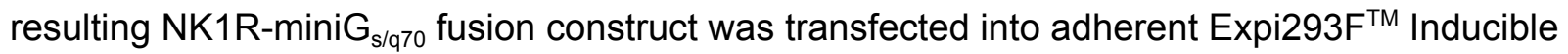
Human Embryonic Kidney Cells (unauthenticated and untested for mycoplasma contamination, Life Technologies) using Lipofectamine $2000^{\odot}$ and cells were maintained in DMEM (Gibco, $11995-065)+10 \%$ FBS (Gibco), $100 \mathrm{U} / \mathrm{mL}$ penicillin and $100 \mu \mathrm{g} / \mathrm{mL}$ streptomycin at $37^{\circ} \mathrm{C}$ and $5 \% \mathrm{CO}_{2}$ in a standing incubator. Cells stably incorporating the $\mathrm{NK} 1 \mathrm{R}-\mathrm{mini}_{\mathrm{s} / \mathrm{q} 70}$ fusion plasmid were selected under antibiotic pressure with zeocin $(500 \mu \mathrm{g} / \mathrm{mL})$ and blasticidin $(10 \mu \mathrm{g} / \mathrm{mL})$. The resulting polyclonal Expi293F ${ }^{\mathrm{TM}} \mathrm{NK} 1 \mathrm{R}-\mathrm{miniG}_{\mathrm{s} / \mathrm{q} 70}$ stable cell line was then adapted to suspension culture and maintained in Expi293 ${ }^{\mathrm{TM}}$ Expression Medium (Gibco) supplemented with zeocin (5 $\mu \mathrm{g} / \mathrm{mL})$ and blasticidin $(5 \mu \mathrm{g} / \mathrm{mL})$ at $37^{\circ} \mathrm{C}$ and $8 \% \mathrm{CO}_{2}$ on a shaking platform at $125 \mathrm{rpm}$. Expression of NK1R-miniG ${ }_{s / q 70}$ fusion protein was induced with addition of $4 \mu \mathrm{g} / \mathrm{mL}$ doxycycline hyclate (Sigma Aldrich) and enhanced with $20 \mathrm{mM}$ sodium butyrate (Sigma Aldrich). Two liters of induced Expi293F ${ }^{\mathrm{TM}}$ NK1R-miniG ${ }_{\mathrm{s} / \mathrm{q} 70}$ stable cells were harvested 24 hours after induction and stored at $-80^{\circ} \mathrm{C}$ until further use.

For purification, cells were thawed and washed with hypotonic buffer (20 mM HEPES pH 7.5, 1 mM EDTA) supplemented with protease inhibitors (20 $\mu \mathrm{g} / \mathrm{mL}$ leupeptin, $160 \mu \mathrm{g} / \mathrm{mL}$ benzamidine, $1 \mathrm{mM}$ PMSF), reducing agent (100 $\mu \mathrm{M}$ TCEP) and $100 \mathrm{nM}$ Substance P (Tocris). The membrane fraction was then solubilized with $50 \mathrm{mM}$ HEPES pH 7.5, $300 \mathrm{mM} \mathrm{NaCl}, 1 \%$ (w/v) lauryl maltose neopentyl glycol (L-MNG, Anatrace), 0.1\% cholesteryl hemisuccinate (CHS, Steraloids), protease inhibitors, $100 \mu \mathrm{M}$ TCEP, $5 \mathrm{mM}$ ATP, $2 \mathrm{mM} \mathrm{MgCl}_{2}$, and $1 \mu \mathrm{M}$ Substance $\mathrm{P}$ for 1.5 hours at $4{ }^{\circ} \mathrm{C}$. After high-speed centrifugation, the supernatant was subjected to affinity purification using homemade M1 anti-FLAG antibody coupled to Sepharose beads. NK1R-miniG ${ }_{s / q 70}$ bound to M1-beads was washed extensively to gradually decrease detergent and salt concentration and was eluted in $50 \mathrm{mM}$ HEPES pH 7.5, $150 \mathrm{mM} \mathrm{NaCl}, 0.0075 \%$ (w/v) L-MNG, 0.0025\% (w/v) glyco-diosgenin (GDN, Anatrace), 0.001\% CHS, $100 \mu \mathrm{M}$ TCEP, 100 nM Substance P, 5 mM EDTA, and $0.2 \mathrm{mg} / \mathrm{mL}$ FLAG peptide (Genscript). Eluted NK1R-miniG $\mathrm{s}_{\mathrm{s} 70}$ was concentrated with 
a $50 \mathrm{kDa}$ MWCO spin concentrator (Millipore) and purified to homogeneity with size-exclusion chromatography, using a Superdex S200 Increase 10/300 GL column (GE Healthcare) equilibrated in $20 \mathrm{mM}$ HEPES pH 7.5, $150 \mathrm{mM} \mathrm{NaCl}, 0.0075 \%$ (w/v) L-MNG, 0.0025\% (w/v) GDN, $0.001 \%$ CHS, $100 \mu \mathrm{M}$ TCEP, and 100 nM Substance P. Fractions containing monodisperse NK1R-miniG $_{\text {s/q70 }}$ fusion protein were pooled, mixed with $2.5 x$ molar excess of $\mathrm{G} \beta_{1} \mathrm{Y}_{2}$ heterodimer, $\mathrm{Nb35}$, and Substance $\mathrm{P}$, and incubated overnight at $4{ }^{\circ} \mathrm{C}$. The next day, the heterotrimeric complex was concentrated with a $50 \mathrm{kDa}$ MWCO spin concentrator and excess $\mathrm{G} \beta_{1} \mathrm{Y}_{2}$ and $\mathrm{Nb} 35$ was removed via size-exclusion chromatography, using a Superdex S200 Increase 10/300 GL column (GE Healthcare) equilibrated in 20 mM HEPES pH 7.5, 150 mM $\mathrm{NaCl}, 0.00075 \%$ (w/v) L-MNG, 0.00025\% (w/v) GDN, 0.0001\% CHS, $100 \mu \mathrm{M}$ TCEP, and $100 \mathrm{nM}$ Substance P. Resulting SP-NK1R-miniG $\mathrm{s}_{\mathrm{s} / \mathrm{70}}$ heterotrimeric complex was concentrated with a 50 kDa MWCO spin concentrator to $1.93 \mathrm{mg} / \mathrm{mL}(14 \mu \mathrm{M})$ for preparation of cryo electron microscopy grids.

\section{Expression and purification of Substance $P(6-11)-N K 1 R-m i n i G_{s / q 70}$ complex}

The SP6-11-bound NK1R-miniG ${ }_{\text {s/q70 }}$ fusion protein was expressed and purified exactly as described above for the full-length SP-NK1R-miniG ${ }_{s / 970}$ fusion protein, with the exception of replacing all SP incubations with SP6-11 throughout the purification. Incubation of SP6-11-NK1R-miniG $\mathrm{s}_{\mathrm{s} 70}$ fusion protein with $\mathrm{G} \beta_{1} \mathrm{Y}_{2}$ and $\mathrm{Nb} 35$ was performed as described above

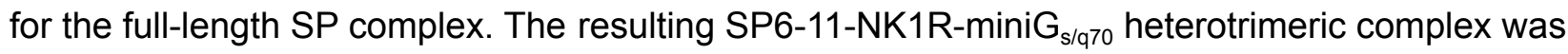
concentrated with a $50 \mathrm{kDa}$ MWCO spin concentrator to $2.91 \mathrm{mg} / \mathrm{mL}(21 \mu \mathrm{M})$ for preparation of cryo electron microscopy grids.

\section{Expression and purification of full-length Substance P-NK1R-miniG ${ }_{\mathrm{s} 399}$ complex}

The NK1R-miniG ${ }_{s / q 70}$ fusion construct (generation described above) was modified to replace the miniG $\mathrm{s}_{\mathrm{s} / \mathrm{q} 70}$ protein with the $\operatorname{miniG}_{\mathrm{s} 399}{ }^{33,34}$ protein using Gibson cloning. The subsequent NK1R-miniG ${ }_{s 399}$ fusion construct was transiently transfected into 200-mLs of Expi293F ${ }^{\mathrm{TM}}$ Inducible Human Embryonic Kidney Cells (unauthenticated and untested for mycoplasma contamination, Life Technologies) using the Expifectamine Transfection Kit (Life Technologies), following the manufacturer's instructions. Expression of the $N K 1 R-m_{\text {iniG }} 399$ fusion protein was induced and enhanced 18 hours after transfection with addition of $1 \mu \mathrm{g} / \mathrm{mL}$ doxycycline hyclate (Sigma Aldrich), $10 \mathrm{mM}$ sodium butyrate (Sigma Aldrich), and addition of enhancers from the 
Expifectamine Transfection Kit, as per the manufacturer's instructions. Cells were harvested 24 hours after induction and stored at $-80{ }^{\circ} \mathrm{C}$ until further use.

The full-length SP-NK1R-miniG ${ }_{s 399}$ fusion protein was purified exactly as described above for the full-length SP-NK1R-miniG ${ }_{s / q 70}$ fusion protein. Incubation of SP-NK1R-miniG ${ }_{s 399}$ fusion protein with $G \beta_{1} Y_{2}$ and $\mathrm{Nb35}$ was performed exactly as described above for the SP-NK1R-miniG $\mathrm{s}_{\mathrm{s} / 0}$ complex. The resulting SP-NK1R-miniG ${ }_{\mathrm{s} 399}$ heterotrimeric complex was concentrated with a 50 kDa MWCO spin concentrator to $2.86 \mathrm{mg} / \mathrm{mL}(20 \mu \mathrm{M})$ for preparation of cryo electron microscopy grids.

\section{Expression and purification of $G \beta_{1} \mathbf{Y}_{2}$}

The $\mathrm{G} \beta_{1} \mathrm{Y}_{2}$ heterodimer was expressed in Trichoplusia ni (Hi5) insect cells (Expression Systems, unauthenticated and untested for mycoplasma contamination) using a single baculovirus, as previously described ${ }^{60}$. A single bicistronic baculovirus encoding the human $G \beta_{1}$ subunit with a $\mathrm{N}$-terminal $6 \mathrm{x}$ His-tag and rhinovirus $3 \mathrm{C}$ protease site and untagged human $\mathrm{Gy}_{2}$ subunit was generated using the BestBac method (Expression systems) in Spodoptera frugiperda (Sf9) insect cells (Expression Systems 94-001F, unauthenticated and untested for mycoplasma contamination). Hi5 insect cells were transduced with baculovirus at a density of $\sim 3.0 \times 10^{6}$ cells $/ \mathrm{mL}$, grown at $27^{\circ} \mathrm{C}$ and shaking at $130 \mathrm{rpm}$. Cultures were harvested 48 hours after transduction, and cell pellets were stored at $-80^{\circ} \mathrm{C}$ until further use. Frozen cell pellets were thawed and washed in a hypotonic buffer containing $10 \mathrm{mM}$ Tris $\mathrm{pH} 8.0,5 \mathrm{mM}$ $\beta$-mercaptoethanol ( $\beta-M E)$, and protease inhibitors $(20 \mu \mathrm{g} / \mathrm{mL}$ leupeptin, $160 \mu \mathrm{g} / \mathrm{mL}$ benzamidine). The membrane fraction was collected by centrifugation and then solubilized with $20 \mathrm{mM}$ HEPES pH 7.5, $100 \mathrm{mM} \mathrm{NaCl}, 1 \%$ (w/v) sodium cholate, $0.05 \%$ dodecyl maltoside (DDM, Anatrace), $0.005 \%$ cholesteryl hemisuccinate (CHS, Steraloids), $5 \mathrm{mM} \beta-\mathrm{ME}$, protease inhibitors, and $5 \mathrm{mM}$ Imidazole for 1 hour at $4{ }^{\circ} \mathrm{C}$. After high-speed centrifugation, the supernatant was subjected to affinity purification with HisPur ${ }^{T M}$ Ni-NTA resin (Thermo Scientific). Bound G $\beta_{1} \mathrm{Y}_{2}$ heterodimer was washed extensively and detergent was slowly exchanged to $0.1 \%(\mathrm{w} / \mathrm{v})$ lauryl maltose neopentyl glycol (L-MNG, Anatrace) and 0.01\% CHS before elution with 20 mM HEPES $\mathrm{pH}$ 7.5, $100 \mathrm{mM} \mathrm{NaCl}, 0.1 \%$ L-MNG, 0.01\% CHS, $270 \mathrm{mM}$ imidazole, $1 \mathrm{mM}$ dithiothreitol (DTT), and protease inhibitors. Eluted $\mathrm{G} \beta_{1} \mathrm{Y}_{2}$ heterodimer was pooled and $3 \mathrm{C}$ protease was added to cleave the $\mathrm{N}$-terminal $6 x$ His-tag. The resulting $G \beta_{1} \mathrm{~V}_{2}$ heterodimer was dialyzed overnight in 20 $\mathrm{mM}$ HEPES pH 7.5, $100 \mathrm{mM} \mathrm{NaCl}, 0.02 \%$ L-MNG, 0.002\% CHS, $1 \mathrm{mM}$ DTT, and $10 \mathrm{mM}$ 
imidazole. Reverse Ni-NTA affinity chromatography was performed to remove uncleaved heterodimer. The resulting $\mathrm{G} \beta_{1} \gamma_{2}$ was then incubated for 1 hour at $4{ }^{\circ} \mathrm{C}$ with lambda phosphatase (New England Biolabs), calf intestinal phosphatase (New England Biolabs), and antarctic phosphatase (New England Biolabs) to dephosphorylate the protein. $G \beta_{1} \gamma_{2}$ was further purified by anion exchange chromatography using a MonoQ4.6/100 PE (GE Healthcare) column. The resulting protein was pooled and dialyzed overnight in $20 \mathrm{mM} \mathrm{HEPES} \mathrm{pH} \mathrm{7.5,} 100 \mathrm{mM} \mathrm{NaCl}$, $0.02 \%$ L-MNG, and $100 \mu \mathrm{M}$ TCEP, concentrated with a $3 \mathrm{kDa}$ centrifugal concentrator. Glycerol was added to a final concentration of $20 \%$, and the protein was flash frozen in liquid $\mathrm{N}_{2}$ and stored at $-80^{\circ} \mathrm{C}$ until further use.

\section{Expression and purification of $\mathrm{Nb} 35$}

Nanobody35 $(\mathrm{Nb} 35)^{26}$ with a N-terminal pelB signal sequence and a C-terminal Protein $\mathrm{C}$ affinity tag (EDQVDPRLIDGK) was cloned into a pET-26b IPTG-inducible bacterial expression vector. This vector was transformed into BL21 Rosetta Escherichia coli cells and grown overnight in Luria Broth supplemented with $50 \mu \mathrm{g} / \mathrm{mL}$ kanamycin shaking at $225 \mathrm{rpm}$ and $37^{\circ} \mathrm{C}$. Next day, the saturated overnight culture was used to inoculate $8 \mathrm{~L}$ of Terrific Broth (supplemented with $0.1 \%$ glucose, $2 \mathrm{mM} \mathrm{MgCl}$, and $50 \mu \mathrm{g} / \mathrm{mL}$ kanamycin) and cells were grown shaking at $225 \mathrm{rpm}$ at 37 ${ }^{\circ} \mathrm{C}$. When cells reached an $\mathrm{OD}_{600}=0.6$, expression of $\mathrm{Nb} 35$ was induced with addition of 400 $\mu \mathrm{M}$ IPTG and the temperature was reduced to $20^{\circ} \mathrm{C}$ for 21 hours. Cells were harvested by centrifugation and stored in the $-80^{\circ} \mathrm{C}$ until further use. For purification of $\mathrm{Nb35}$, cells were thawed and resuspended in SET Buffer (200 mM Tris pH 8.0, $500 \mathrm{mM}$ sucrose, $0.5 \mathrm{mM}$ EDTA) supplemented with protease inhibitors (20 $\mathrm{gg} / \mathrm{mL}$ leupeptin, $160 \mu \mathrm{g} / \mathrm{mL}$ benzamidine) and benzonase. After 30 minutes of stirring, two equal volumes of miliQ $\mathrm{H}_{2} \mathrm{O}$ were added to initiate hypotonic lysis. After 45 minutes of stirring, $\mathrm{NaCl}$ was added to $150 \mathrm{mM}, \mathrm{CaCl} 2$ was added to 2 $\mathrm{mM}$, and $\mathrm{MgCl} 2$ was added to $2 \mathrm{mM}$. Insoluble matter was then separated by high speed centrifugation and the supernatant was subjected to affinity purification with homemade anti-Protein $\mathrm{C}$ antibody coupled to Sepharose beads. After extensive washing, bound Nb35 was eluted with $20 \mathrm{mM}$ HEPES pH 7.5, $100 \mathrm{mM} \mathrm{NaCl}$, and $2 \mathrm{mM} \mathrm{CaCl}$, $0.2 \mathrm{mg} / \mathrm{mL}$ Protein C peptide, and $5 \mathrm{mM}$ EDTA pH 8.0. Eluted Nb35 was collected, concentrated, and injected over an Superdex S75 Increase 10/300 GL column (GE Healthcare) size-exclusion chromatography column equilibrated in $20 \mathrm{mM}$ HEPES pH 7.5, $100 \mathrm{mM} \mathrm{NaCl}$. Fractions containing Nb35 were collected, concentrated, glycerol was added to a final concentration of $20 \%$, and aliquots of Nb35 were flash frozen in liquid nitrogen and stored in the $-80{ }^{\circ} \mathrm{C}$ until further use. 


\section{Cryo-EM sample vitrification and data collection}

For cryogenic electron microscopy, $3 \mu \mathrm{L}$ of the SP-NK1R-miniG $\mathrm{s}_{\mathrm{s} / \mathrm{7} 0}$ heterotrimeric complex at 13.8 $\mu \mathrm{M}$ was added to 300 Mesh 1.2/1.3R Au Quantifoil grids previously glow discharged at 15 $\mathrm{mA}$ for 30 seconds with a Pelco easiGlow Glow discharge cleaning system. Grids were blotted with Whatman No. 1 qualitative filter paper in a Vitrobot Mark IV (Thermo Fisher) at $8^{\circ} \mathrm{C}$ and $100 \%$ humidity for 1 second using a blot force of 4 prior to plunging into liquid ethane. The SP-NK1R-miniG ${ }_{\mathrm{s} 399}$ and SP6-11-NK1R-miniG $\mathrm{s} / \mathrm{q} 70$ heterotrimeric complexes were frozen under identical blotting conditions at $20 \mu \mathrm{M}$ and $20.7 \mu \mathrm{M}$, respectively.

For the SP-NK1R-miniG $\mathrm{s}_{\mathrm{s} / \mathrm{70}}$ heterotrimeric complex, 3,755 super-resolution movies were recorded with a $300 \mathrm{keV}$ Titan Krios (Thermo Fisher) equipped with a K3 detector and BioQuantum energy filter (Gatan) with a zero-loss energy selection slit width set to $20 \mathrm{eV}$ and a defocus range of -0.8 to $-2.0 \mu \mathrm{m}$. Each dose-fractionated 120 -frame movie was collected at a dose rate of $8.0 \mathrm{e}^{-p i x}{ }^{-1} \mathrm{~s}^{-1}$ for 5.9 seconds at a nominal magnification of 105,000x (physical pixel size of $0.835 \AA$ pix $^{-1}$ ) resulting in a cumulative dose of $67 \mathrm{e}^{-} \AA^{-2}$. Exposure areas were acquired with automated scripts in a $3 \times 3$ image shift collection strategy using SerialEM. ${ }^{61}$ The 3878

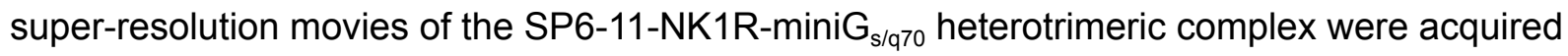
with identical acquisition settings on the same $300 \mathrm{keV}$ Titan Krios as previously described.

For the SP-NK1R-miniG ${ }_{s 399}$ heterotrimeric complex, 3670 dose-fractionated movies were collected in counting mode with a $300 \mathrm{keV}$ Titan Krios equipped with a K3 detector and BioQuantum energy filter (Gatan) with a zero-loss energy selection slit width set to $20 \mathrm{eV}$ and a

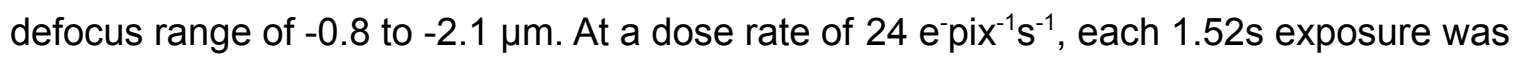
fractionated across 60 frames for a total dose of $49.4 \mathrm{e}^{-} \AA^{-2}$. Data were collected using aberration free image shift (AFIS) with EPU 2.10.

\section{Cryo-EM Image Processing}

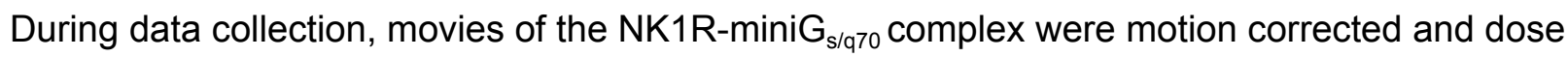
weighted with UCSF MotionCor2 ${ }^{62}$ and binned to physical pixel size. Post-acquisition, micrographs were imported into cryoSPARC ${ }^{63}$ for contrast transfer function determination via patch CTF. 7,329,811 particles were template picked with $20 \AA$ low-pass filtered projections of the NK1R-miniG ${ }_{s / q 70}$ heterotrimeric complex from a prior screening collection on a $200 \mathrm{keV}$ Talos 
Arctica. 3,555 micrographs comprising 6,945,760 particles were curated for further processing via CTF fit estimated resolution, ice thickness, and particle pick power scores. Particles were extracted in a 72-pixel box, Fourier cropped from 288 pixels. 200 2D class averages were generated using a maximum alignment resolution of $8 \AA, 30$ online-EM iterations, and 200 particles per class during each online EM iteration. 1,901,254 particles were selected from qualitatively "good" classes containing any averages that showed "multi-lobed" densities or appeared to be "top" or "bottom" views. Following 2D classification, 500,000 selected particles were used for ab initio reconstruction into 3 classes. A single "multi-lobed" class suggestive of an intact NK1R-miniG s/q70 $_{\text {n }}$ heterotrimeric complex was selected and used alongside three poorly aligned "junk" classes generated from ab initio volumes from $<100$ particles. All particles selected from 2D classification as described above were subject to 3D classification with alignment against the NK1R-miniG ${ }_{s / q 70}$ and poorly aligned classes from the earlier ab initio runs. 718,386 particles in the NK1R complex class were re-extracted without Fourier cropping and subject to the same 3D classification scheme with a new ab initio NK1R-miniG s/q70 $_{\text {neterimeric }}$ complex class generated from 300,000 particles and three "junk" classes. Using UCSF pyEM ${ }^{64}$, 589,973 particles in the NK1R-miniG ${ }_{\mathrm{s} / \mathrm{q} 70}$ class were exported from cryoSPARC for alignment-free classification in RELION ${ }^{65}$ into 4 classes for 50 iterations with a $\tau$ parameter of 8 . Two classes containing 122,222 particles were imported into cisTEM ${ }^{66}$ for manual "focused" refinements utilizing whole complex and upper-transmembrane domain masks, respectively. To

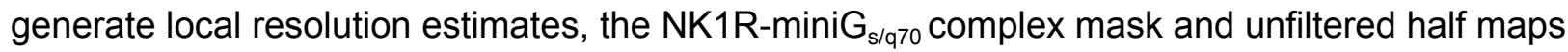
were imported into cryoSPARC. The same half maps and mask were used for directional FSC curve generation $^{67}$.

3,670 SP-NK1R-miniG ${ }_{\mathrm{s} 399}$ movies were motion-corrected post acquisition with UCSF MotionCor2. CTF estimation and template-based automatic particle picking were performed in cryoSPARC. 4,865,341 particles were picked using templates generated from 2D class averages of SP-NK1R-miniG ${ }_{\mathrm{s} 399}$ as determined from an earlier screening collection. 4,256,322 particles were extracted in an 80 pixel box after curating micrographs via CTF fit estimated resolution, ice thickness, and particle pick power scores. Extracted particles were subject to a round of 3D classification with alignment (Heterogeneous refinement) using a $20 \AA$ low-pass

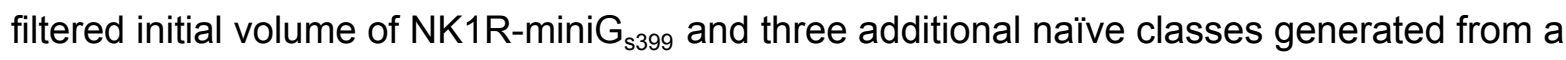
deliberately under-sampled $A b$ initio job. Particles classified into the $\mathrm{NK}_{1 \mathrm{R}-\mathrm{mini}} \mathrm{s}_{\mathrm{s} 99}$ class were selected for further workup. This process was repeated over two additional rounds, decreasing 
particle Fourier cropping at each subsequent extraction round. 561,901 unbinned particles

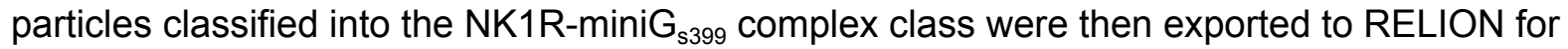
alignment-free classification on only 7TM domain features for 25 iterations, 4 classes, $\tau=8.288,659$ particles in three classes were imported into cisTEM for manual "focused" refinements as in the NK1R-miniG ${ }_{\text {s/q70 }}$ processing.

3,878 motion-corrected, dose-weighted SP6-11-NK1R-miniG ${ }_{\text {s/q70 }}$ complex sums were imported into cryoSPARC for CTF estimation and template-based autopicking as previously described. $4,135,538$ particles were picked with templates generated from 2D classes determined from a prior screening dataset. Particles were extracted in a 72-pixel box and underwent iterative rounds of 3D classification with alignment on successively unbinned particles classified into a SP6-11-NK1R-miniG ${ }_{\text {s/q70 }}$ complex class. 553,506 particles were exported to RELION for alignment-free classification on the 7TM domain using the same parameters as previously described, though with six classes instead of four. Visual inspection of each class led to selection of a single class generated from 59,926 particles for manual "focused" refinements in cisTEM using the same masking scheme as for the previously described complexes. Half maps and masks were re-imported into cryoSPARC for GS-FSC determination. dFSC curve calculation utilized the same half maps and masks from focused refinement.

\section{Model building and refinement: SP-NK1R-miniG $\mathrm{s}_{\mathrm{s} 70}$}

The initial model for NK1R was taken from the high-resolution $(2.2 \AA)$ inactive-state structure of NK1R bound to the clinically approved antagonist, netupitant (PDB: 6HLP) ${ }^{38}$. The model was docked into the $3.0 \AA$ EM density map using manual adjustment and the 'fit in map' function in UCSF ChimeraX ${ }^{68}$. The initial model was manually rebuilt in Coot and refined with both iterative adjustment in Coot and multiple rounds of global minimization and real space refinement using the Phenix.real_space_refine tool in Phenix ${ }^{69,70}$. In areas of weak sidechain density, residues were capped at the $C \beta$ position to retain sequence information; in areas of weak mainchain density, residues were truncated from the final model. This process was repeated to model the miniG $\mathrm{s}_{\mathrm{s} / 70}$ subunit (starting model: miniG $_{\mathrm{s}}$ subunit, PDB: $6 \mathrm{LI} 3^{30}$ ), G $\beta_{1} \mathrm{Y}_{2}$ (starting model: G $\beta_{1} \mathrm{Y}_{2}$ heterodimer, PDB: $3 S N 6{ }^{26}$ ), and Nb35 (starting model: Nb35, PDB: 3SN6 ${ }^{26}$ ). Models were combined into one PDB file in Coot and the model geometry was assessed using Molprobity ${ }^{71}$. Further validation was performed with EMRinger ${ }^{72}$ to compare the map to the model. Finally, 
map-to-model FSCs were calculated in Phenix ${ }^{73}$. All structure figures were prepared with PyMOL or UCSF ChimeraX ${ }^{68}$.

To model the Substance $\mathrm{P}$ peptide, all peptide residues were manually built into the Substance $\mathrm{P}$ EM density in Coot. The resulting Substance $P$ model was refined with iterative adjustment in Coot and multiple rounds of global minimization and real space refinement using the real_space_refine tool in Phenix. Due to weak side chain density, R1 and K3 of the peptide are capped at the $C \beta$ position of the residue. To build the amidated C-terminus of $M 11$, a peptide bond connecting the carbonyl-carbon of M11 to a new nitrogen atom was created in Coot. Bond-lengths, bond-angles, dihedral angles, and planes for the new amide moiety were manually adjusted to reflect the accepted values for amide moieties in protein structures.

\section{Model building and refinement: SP-NK1R-miniG ${ }_{\mathrm{s} 399}$ \& SP6-11-NK1R-miniGs/q70 complexes} The SP-NK1R-miniG $\mathrm{s}_{\mathrm{s} / 0}$ complex model was docked into the $3.1 \AA$ and $3.2 \AA$ EM density maps for the SP-NK1R-miniG ${ }_{\mathrm{s} 399}$ and SP6-11-NK1R-miniGs/q70, respectively. The models were built and refined as described above. Models were combined into one PDB file in Coot and the model geometry was assessed using Molprobity ${ }^{71}$. Further validation was performed with EMRinger ${ }^{72}$ to compare the map to the model. Finally, map-to-model FSCs were calculated in Phenix ${ }^{73}$. All structure figures were prepared with PyMOL or UCSF ChimeraX ${ }^{68}$.

\section{Generation of Stable Cell lines and Transfection for signaling assays.}

Flp-In-HEK293 cells were grown in DMEM supplemented with $5 \%$ fetal bovine serum and maintained at $37{ }^{\circ} \mathrm{C}$ in a humidified incubator containing $5 \% \mathrm{CO} 2$. Flp-In-HEK cells were transfected with the pOG44 vector encoding Flp recombinase and the pcDNA5 vector encoding the $\mathrm{NK}_{1} \mathrm{R}$ at a ratio of 9:1 using lipofectamine as the transfection reagent. Twenty-four hours after transfection, the cells were subcultured and forty-eight hours later, the medium was supplemented with $200 \mu \mathrm{g} / \mathrm{ml}$ Hygromycin B as selection agent, to obtain cells stably expressing the $\mathrm{NK}_{1} \mathrm{R}$.

\section{Intracellular $\mathrm{Ca}^{2+}$ mobilization signaling assay}

FIp-In-HEK293 cells stably expressing human $\mathrm{NK}_{1} \mathrm{R}$ WT or mutants were plated in Poly-D-Lysine coated 96-well plates. Cells were washed with calcium buffer (10 mM HEPES, $150 \mathrm{mM} \mathrm{NaCl}, 2.2 \mathrm{mM} \mathrm{CaCl}_{2}, 1.18 \mathrm{mM} \mathrm{MgCl}_{2}, 2.6 \mathrm{mM} \mathrm{KCl}, 10 \mathrm{mM}$ D-glucose, 0.5\% w/v BSA, 4 
$\mathrm{mM}$ probenecid, $0.05 \%$ v/v pluronic acid F127; $\mathrm{pH} 7.4$ ) and then loaded with $1 \mu \mathrm{M}$ Fura-2 AM ester (Life Technologies) in calcium buffer for $45 \mathrm{~min}$ at $37^{\circ} \mathrm{C}$. Calcium mobilization was measured using a FlexStation 3 plate reader (Molecular Devices). Fluorescence (excitation: 340 $\mathrm{nm}$ and $380 \mathrm{~nm}$; emission: $520 \mathrm{~nm}$ ) was measured at $4 \mathrm{~s}$ intervals for 5 cycles. After establishing baseline fluorescence, cells were stimulated with increasing concentrations of the agonists or $1 \mu \mathrm{M}$ ionomycin (positive control for normalization, to obtain a receptor-independent response) and the response was measured for 17 cycles in SoftMax Pro (v5.4.4) software. GraphPad Prism software (v. 9.0) was used to calculate the area under the curve from the kinetic data and for normalization to vehicle and positive control.

\section{cAMP accumulation signaling assay}

Flp-In-HEK293 cells stably expressing the human $\mathrm{NK}_{1} \mathrm{R}$ WT or mutants were seeded at a density of 2,000,000 cells per 10-cm dish and were transfected the following day using polyethylenimine as the transfection reagent. The cells were transfected with $5 \mu \mathrm{g}$ CAMYEL biosensor (cAMP sensor using YFP-Epac-RLuc), to allow the detection of cAMP levels by Bioluminescence Resonance Energy Transfer. Twenty-four hours after transfection, the cells were plated into Poly-D-Lysine coated 96-well CulturPlates (PerkinElmer) and grown overnight. The cells were equilibrated in Hank's balanced salt solution at $37^{\circ} \mathrm{C}$ before starting the experiment. Coelenterazine (Promega) was added at a final concentration of $5 \mu \mathrm{M}$ at least 3 min before measurement. After establishing a baseline response, cells were stimulated with increasing concentrations of the agonists or $10 \mu \mathrm{M}$ forskolin (positive control for normalization, to obtain a receptor-independent response) and the response was measured for a total of 30 min. The signals were detected at 445-505 nm and 505-565 nm using a LUMIstar Omega instrument (BMG LabTech). GraphPad Prism software (v. 9.0) was used to calculate the area under the curve from the kinetic data and for normalization to vehicle and positive control.

\section{IP1 accumulation signaling assay}

Flp-In-HEK293 cells stably expressing the human $\mathrm{NK}_{1} \mathrm{R}$ WT or mutants were plated in Poly-D-Lysine coated 96-well plates overnight. Cells were equilibrated in Cisbio Bioassays' IP-One Gq kit stimulation buffer (10 mM HEPES, $146 \mathrm{mM} \mathrm{NaCl}, 4.2 \mathrm{mM} \mathrm{KCl}, 0.5 \mathrm{mM} \mathrm{MgCl}_{2}, 1$ $\mathrm{mM} \mathrm{CaCl}_{2}, 5.5 \mathrm{mM}$ D-Glucose, $50 \mathrm{mM} \mathrm{LiCl}, \mathrm{pH}$ 7.4) for 1 hour prior to agonist stimulation for 1 hour at $37^{\circ} \mathrm{C}$. Cells were then lysed in $25 \mu$ l of lysis buffer (50 mM HEPES, $15 \mathrm{mM} \mathrm{KF}, 1.5 \%$ $(\mathrm{v} / \mathrm{v})$ Triton-X-100, 3\% (v/v) foetal bovine serum, $0.2 \%(\mathrm{w} / \mathrm{v})$ bovine serum albumin, $\mathrm{pH} 7.0)$ and 
$14 \mu \mathrm{l}$ of lysis were added to wells of a 384 well white proxiplate (PerkinElmer) for analysis. The Cisbio Bioassays' IP-One competitive immunoassay kit was used to measure myo-Inositol 1 phosphate (IP1) accumulation in cells, based on HTRF® fluorescence resonance energy transfer (FRET) between d2-labeled IP1 (acceptor) and anti-IP1-Cryptate (donor) antibody. These reagents were diluted 1:20 in the lysis buffer and $3 \mu$ of each was added to each well containing the lysates. Lysates were incubated for 1 hour at room temperature before FRET was detected using an Envision plate reader (PerkinEImer). Emission of Lumi4TM-Tb cryptate was detected at $620 \mathrm{~nm}$ and emission of d2-conjugated IP1 at $665 \mathrm{~nm}$. Results were calculated from the $665 \mathrm{~nm} / 620 \mathrm{~nm}$ ratio, since the specific signal is inversely proportional to the concentration of IP1, data were transformed and normalised to $1 \mu \mathrm{M}$ SP in GraphPad Prism software (v. 9.0).

\section{Enzyme-linked immunosorbent assay (ELISA)}

Flp-In-HEK293 cells stably expressing human Flag-NK1R WT, mutants, or untransfected as a control were plated into poly-D-lysine-coated 48-well plates and allowed to adhere overnight. Cells were fixed with $3.7 \%(\mathrm{v} / \mathrm{v})$ paraformaldehyde in tris-buffered saline (TBS) for $30 \mathrm{~min}$. For total expression, cells were permeabilized by 30 -min incubation with $0.5 \%$ (v/v) NP-40 in TBS. Cells were then incubated in blocking buffer $\left[1 \%(\mathrm{w} / \mathrm{v})\right.$ skim milk powder in $\left.0.1 \mathrm{M} \mathrm{NaHCO}_{3}\right]$ for 4 hours at room temperature and incubated with mouse M2 anti-FLAG antibody (1:2000, overnight at $4^{\circ} \mathrm{C}$ ). After washing three times with TBS, cells were incubated with anti-mouse horseradish peroxidase-conjugated antibody $(1: 2000)$ for 2 hours at room temperature. Cells were washed and stained using the SIGMAFAST OPD substrate (Sigma-Aldrich). Absorbance at $490 \mathrm{~nm}$ was measured using an EnVision Multilabel Reader (PerkinElmer). Data were normalized to intact HEK293 cells transfected with NK1R WT.

\section{Quantification and Statistical Analysis}

GraphPad Prism software (v. 9.0) was used for signaling data and statistical analysis. Data points are presented as mean \pm standard error of the mean (s.e.m.) based on at least 3 biologically independent experiments with the precise number indicated in Supplementary Table 2 and 3. Data points were normalized to vehicle as $0 \%$ and positive control $(1 \mu \mathrm{M}$ ionomycin or $1 \mu \mathrm{M}$ forskolin) as $100 \%$ unless otherwise stated. Concentration-response curves were fitted using the three parameter log(agonist) vs. response equation. pEC50 values were extracted from the curve fit of each individual experiment. Emax was calculated by subtracting Bottom 
from Top from the curve fit of each individual experiment. Statistical analysis of pEC50 and Emax values was performed using one-way analysis of variance (ANOVA) with Dunnett's multiple comparison-corrected post hoc test against NK1R WT SP or NK1R WT SP6-11 unless otherwise stated.

\section{Molecular Dynamics: System setup}

We performed simulations of NK1R bound to full-length substance $P(S P)$ and a truncated version of SP reduced to the C-terminal residues 6-11 (SP6-11). These simulations were initiated from the cryo-EM structure of SP-bound NK1R, with the intracellular G protein removed and, for SP6-11-bound simulations, residues 1-5 of SP removed.

The peptide-bound NK1R structure was prepared for simulation with Maestro (Schrödinger Release 2019-4: Maestro, Schrödinger, LLC, New York, NY, 2019). Missing amino acid side chains were modeled using Prime ${ }^{74,75}$. Residues 226-237 are missing in the cryo-EM structure and were not modeled in. Neutral acetyl and methylamide groups were added to cap the $\mathrm{N}$ - and C-termini, respectively, of the NK1R protein chains. The N-termini of SP and SP6-11 were prepared in their charged form, while the C-termini were amidated. Titratable residues were kept in their dominant protonation state at pH 7, except for E2.50 (E78) and D3.49 (D129), which were protonated to their neutral form, as studies indicate that these conserved residues are protonated in active-state class-A GPCRs ${ }^{76,77}$. Histidine residues were modeled as neutral, with a hydrogen atom bound to either the delta or epsilon nitrogen depending on which tautomeric state optimized the local hydrogen-bonding network. Dowser ${ }^{78}$ was used to add water molecules to protein cavities, and the protein structures were aligned on the transmembrane (TM) helices of the inactive NK1R crystal structure (PDB ID: 6HLP) ${ }^{38}$ in the Orientation of Proteins in Membranes (OPM) database ${ }^{79}$. The aligned structures were inserted into a pre-equilibrated palmitoyloleoyl-phosphatidylcholine (POPC) membrane bilayer using Dabble ${ }^{80}$. Sodium and chloride ions were added to neutralize each system at a concentration of $150 \mathrm{mM}$. The final systems comprised 57605-58494 atoms, including 134 lipid molecules and 11565-11831 water molecules. Approximate system dimensions were $80 \AA$ x $80 \AA$ x $94 \AA$.

\section{Molecular Dynamics: Simulation protocols}

For each simulation condition (SP-bound and SP6-11-bound), we performed 12 independent simulations ( 2- $\mu$ s each) in which initial atom velocities were assigned randomly and 
independently. We employed the CHARMM36m force field for protein molecules, the CHARMM36 parameter set for lipid molecules and salt ions, and the associated CHARMM TIP3P model for water ${ }^{81-83}$. Simulations were run using the AMBER18 software ${ }^{84}$ under periodic boundary conditions with the Compute Unified Device Architecture (CUDA) version of Particle-Mesh Ewald Molecular Dynamics (PMEMD) on one GPU ${ }^{85}$.

After energy minimization, the systems were first heated over $12.5 \mathrm{ps}$ from $0 \mathrm{~K}$ to $100 \mathrm{~K}$ in the NVT ensemble using a Langevin thermostat with harmonic restraints of $10.0 \mathrm{kcal} \cdot \mathrm{mol}^{-1} \cdot \AA^{-2}$ on the non-hydrogen atoms of the lipids, protein, and ligand. Initial velocities were sampled from a Boltzmann distribution. The systems were then heated to $310 \mathrm{~K}$ over $125 \mathrm{ps}$ in the NPT ensemble. Equilibration was performed at $310 \mathrm{~K}$ and 1 bar in the NPT ensemble, with harmonic restraints on the protein and ligand non-hydrogen atoms tapered off by $1.0 \mathrm{kcal} \cdot \mathrm{mol}^{-1} \cdot \AA^{-2}$ starting at $5.0 \mathrm{kcal} \cdot \mathrm{mol}^{-1} \cdot \AA^{-2}$ in a stepwise manner every $2 \mathrm{~ns}$ for $10 \mathrm{~ns}$, and finally by $0.1 \mathrm{kcal} \cdot \mathrm{mol}^{-1} \cdot \AA^{-2}$ every 2 ns for an additional 18 ns. All restraints were completely removed during production simulation. Production simulations were performed at $310 \mathrm{~K}$ and $1 \mathrm{bar}$ in the NPT ensemble using the Langevin thermostat and Monte Carlo barostat. The simulations were performed using a timestep of $4.0 \mathrm{fs}$ while employing hydrogen mass repartitioning ${ }^{86}$. Lengths of bonds to hydrogen atoms were constrained using $\mathrm{SHAKE}^{87}$. Non-bonded interactions were cut off at 9.0 $\AA$, and long-range electrostatic interactions were calculated using the particle-mesh Ewald (PME) method with an Ewald coefficient ( $\beta$ ) of approximately $0.31 \AA$ and B-spline interpolation of order 4 . The PME grid size was chosen such that the width of a grid cell was approximately 1 $\AA$.

\section{Molecular Dynamics: Simulation analysis protocols}

The AmberTools17 CPPTRAJ package ${ }^{88}$ was used to reimage trajectories, while Visual Molecular Dynamics (VMD) ${ }^{89}$ and PyMOL (The PyMOL Molecular Graphics System, Version 2.0 Schrödinger, LLC.) were used for visualization. MDAnalysis ${ }^{90}$ was used for simulation analysis.

Root mean square fluctuation (RMSF) values shown in Figure 4B measure the extent to which a group of atoms fluctuates around its average position in simulation and is thus a measure for mobility. The first 500 ns of each simulation trajectory were omitted from this analysis to avoid including any initial relaxation of the system in the measurement. The analysis was performed on 1501 frames per simulation, where each frame is separated by 1 ns. For each simulation, an 
average position of each atom in a specified group (residues 6-11, F7, or M11; for each of these three groups all atoms were included) was calculated. Then, the RMSF was obtained as the time-average of the RMSD to the average structure for each simulation. For the RMSF of residues 6-11, trajectories were aligned to the initial cryo-EM structure on all transmembrane helix $\mathrm{C} \alpha$ atoms. For the RMSF of residues F7 and M11, trajectories were aligned to the initial cryo-EM structure on all Ca atoms of residues 6-11 of SP to better capture the individual residue movement independent of the overall movement of the entire peptide. Each bar in Figure 4 represents the mean RMSF value over all 12 simulations per condition (either SP or SP6-11) and the error bars denote the standard error of the mean. To test statistical significance, we performed two-sided t-tests of unequal variance (Welch's t-tests).

For each of the 3 renderings in Figure 4A and for each of the 3 renderings in Figure 4C, we chose 10 representative simulation frames illustrating the dynamics of the backbone of residues 6-11 and the side chains of F7 and M11.

\section{Data Availability}

All data generated or analyzed during this study are included in this published article and its Supplementary Information. Coordinates for SP-NK1R-miniG ${ }_{s / q 70}$, SP-NK1R-miniG ${ }_{s 399}$, and the SP6-11-NK1R-miniG $\mathrm{s} / \mathrm{q} 70$ complex have been deposited in the Protein Data Bank under accession codes $X X X X, X X X X, X X X X$, respectively. Unsharpened cryo-EM maps for

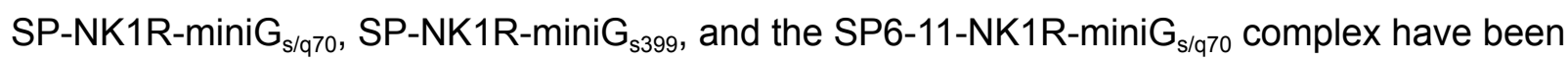
deposited in the Electron Microscopy Data Bank under accession codes XXXXX, XXXXX, and XXXXX, respectively. Sharpened cryo-EM maps for SP-NK1R-miniG ${ }_{\mathrm{s} / \mathrm{q} 70}$, SP-NK1R-miniG $\mathrm{s}_{\mathrm{s} 399}$, and the SP6-11-NK1R-miniG ${ }_{s / 970}$ complex have been deposited in the Electron Microscopy Data Bank under accession codes $X X X X X, X X X X X$, and $X X X X X$, respectively.

\section{Acknowledgements}

This work was supported by National Institutes of Health (NIH) grants DP5OD023048 (A.M.), R01GM127359 (R.O.D.), and a National Health and Medical Research Council (NHMRC) Project Grant APP1138448 (D.M.T), NHMRC Investigator Grant APP1196951 (D.M.T), Australian Research Council DECRA grant DE170100152 (D.M.T.), and Australian Research Council Centre of Excellence in Convergent Bio-Nano Science and Technology (N.A.V). This material is based upon work supported by the National Science Foundation Graduate Research 
Fellowship Program (J.A.H.) under Grant No. 2034836. Any opinions, findings, and conclusions or recommendations expressed in this material are those of the author(s) and do not necessarily reflect the views of the National Science Foundation. C.-M.S. was funded by the Human Frontier Science Program (LT000916/2018-L). Cryo-EM equipment at UCSF is partially supported by NIH grants S10OD020054 and S100D021741. Some of this work was performed at the Stanford-SLAC Cryo-EM Center (S2C2), which is supported by the National Institutes of Health Common Fund Transformative High-Resolution Cryo-Electron Microscopy program (U24 GM129541). The content is solely the responsibility of the authors and does not necessarily represent the official views of the National Institutes of Health. Y.C. is an Investigator of Howard Hughes Medical Institute. A.M. acknowledges support from the Pew Charitable Trusts, the Esther and A. \& Joseph Klingenstein Fund and the Searle Scholars Program.

\section{Author Contributions}

J.A.H. purified NK1R-constructs, $G \beta_{1} \gamma_{2}$, and Nb35, established biochemical approaches to reconstitute a NK1R-miniG protein complex, and generated all NK1R mutants. B.F. purified $\mathrm{G} \beta_{1} \bigvee_{2}$, and $\mathrm{Nb35}$, prepared samples for cryo-EM, identified optimal freezing conditions for cryo-EM, screened samples by cryo-EM, collected cryo-EM data, and determined high resolution cryo-EM maps by extensive image processing under the guidance of A.M. and Y.C.. J.A.H. built and refined models of NK1R-miniG protein complexes with input from B.F. and A.M.. A.B.G. generated NK1R stable cell lines and performed cellular signaling experiments under the guidance of N.A.V. and D.M.T.. M.A.D. and C.-M.S. performed and analyzed molecular dynamics simulations under the guidance of R.O.D. The manuscript was written by J.A.H., B.F., A.B.G., M.A.D., C.-M.S., R.O.D, and A.M., with edits from N.A.V., D.M.T., and Y.C. and with approval from all authors. The overall project was supervised by A.M.

\section{Competing Interests}

Research in N.A.V.'s laboratory is funded, in part, by Takeda Pharmaceuticals and Endosome Therapeutics. 


\section{REFERENCES}

1. Steinhoff, M. S., Mentzer, B. von, Geppetti, P., Pothoulakis, C. \& Bunnett, N. W. Tachykinins and Their Receptors: Contributions to Physiological Control and the Mechanisms of Disease. Physiol. Rev. 94, 265-301 (2014).

2. Severini, C., Improta, G., Falconieri-Erspamer, G., Salvadori, S. \& Erspamer, V. The tachykinin peptide family. Pharmacol. Rev. 54, 285-322 (2002).

3. Garcia-Recio, S. \& Gascón, P. Biological and Pharmacological Aspects of the NK1-Receptor. Biomed Res. Int. 2015, 495704 (2015).

4. Laszlo, A. \& Fox, A. J. NK1 receptor antagonists - are they really without effect in the pain clinic? Trends Pharmacol. Sci. 21, 462-464 (2000).

5. Kramer, M. S. et al. Distinct Mechanism for Antidepressant Activity by Blockade of Central Substance P Receptors. Science 281, 1640-1645 (1998).

6. Hargreaves, R. et al. Development of aprepitant, the first neurokinin-1 receptor antagonist for the prevention of chemotherapy-induced nausea and vomiting. Ann. N. Y. Acad. Sci. 1222, 40-48 (2011).

7. Diemunsch, P. et al. Antiemetic activity of the NK1 receptor antagonist GR205171 in the treatment of established postoperative nausea and vomiting after major gynaecological surgery. BJA: British Journal of Anaesthesia 82, 274-276 (1999).

8. Maggi, C. A. The mammalian tachykinin receptors. Gen. Pharmacol. 26, 911-944 (1995).

9. Fong, T. M., Huang, R. R. \& Strader, C. D. Localization of agonist and antagonist binding domains of the human neurokinin-1 receptor. J. Biol. Chem. 267, 25664-25667 (1992).

10. Schwyzer, R. ACTH: A SHORT INTRODUCTORY REVIEW. Ann. N. Y. Acad. Sci. 297, 3-26 (1977).

11. Chavkin, C. \& Goldstein, A. Specific receptor for the opioid peptide dynorphin: 
structure--activity relationships. PNAS 78, 6543-6547 (1981).

12. Portoghese, P. S., Sultana, M., Nagase, H. \& Takemori, A. E. Application of the message-address concept in the design of highly potent and selective non-peptide .delta. opioid receptor antagonists. J. Med. Chem. 31, 281-282 (1988).

13. Hökfelt, T., Pernow, B. \& Wahren, J. Substance P: A Pioneer Amongst Neuropeptides. J. Intern. Med. 27-40 (2001).

14. Mazzone, S. B. \& Geraghty, D. P. Respiratory actions of tachykinins in the nucleus of the solitary tract: characterization of receptors using selective agonists and antagonists. $\mathrm{Br}$. J. Pharmacol. 129, 1121-1131 (2000).

15. Kaczyńska, K., Jampolska, M. \& Szereda-Przestaszewska, M. The role of vagal pathway and NK1 and NK2 receptors in cardiovascular and respiratory effects of neurokinin A. Clin. Exp. Pharmacol. Physiol. 43, 818-824 (2016).

16. Trafton, J. A., Abbadie, C. \& Basbaum, A. I. Differential contribution of substance P and neurokinin A to spinal cord neurokinin-1 receptor signaling in the rat. J. Neurosci. 21, 3656-3664 (2001).

17. Sagan, S., Chassaing, G., Pradier, L. \& Lavielle, S. Tachykinin Peptides Affect Differently the Second Messenger Pathways after Binding to CHO Expressed Human NK1 Receptors. JPET 276, 1039-1048 (1996).

18. Riitano, D., Werge, T. M. \& Costa, T. A mutation changes ligand selectivity and transmembrane signaling preference of the neurokinin-1 receptor. J. Biol. Chem. 272 , 7646-7655 (1997).

19. Evans, T. W., Dixon, C. M., Clarke, B., Conradson, T. B. \& Barnes, P. J. Comparison of neurokinin A and substance P on cardiovascular and airway function in man. Br. J. Clin. Pharmacol. 25, 273-275 (1988). 
20. Joos, G., Pauwels, R. \& van der Straeten, M. Effect of inhaled substance P and neurokinin A on the airways of normal and asthmatic subjects. Thorax 42, 779-783 (1987).

21. Ricciardolo, F. L. et al. Role of nitric oxide and septide-insensitive NK(1) receptors in bronchoconstriction induced by aerosolised neurokinin A in guinea-pigs. Br. J. Pharmacol. 129, 915-920 (2000).

22. Nakajima, Y., Tsuchida, K., Negishi, M., Ito, S. \& Nakanishi, S. Direct linkage of three tachykinin receptors to stimulation of both phosphatidylinositol hydrolysis and cyclic AMP cascades in transfected Chinese hamster ovary cells. J. Biol. Chem. 267, 2437-2442 (1992).

23. Hastrup, H. \& Schwartz, T. W. Septide and neurokinin A are high-affinity ligands on the NK-1 receptor: evidence from homologous versus heterologous binding analysis. FEBS Lett. 399, 264-266 (1996).

24. Ciucci, A., Palma, C., Manzini, S. \& Werge, T. M. Point mutation increases a form of the NK1 receptor with high affinity for neurokinin A and B and septide. Br. J. Pharmacol. 125, 393-401 (1998).

25. Nehmé, R. et al. Mini-G proteins: Novel tools for studying GPCRs in their active conformation. PLoS One 12, e0175642 (2017).

26. Rasmussen, S. G. F. et al. Crystal structure of the $\beta 2$ adrenergic receptor-Gs protein complex. Nature 477, 549-555 (2011).

27. Ballesteros, J. A. \& Weinstein, H. Chapter 19: Integrated methods for the construction of three-dimensional models and computational probing of structure-function relations in $\mathrm{G}$ protein-coupled receptors. in Receptor Molecular Biology (ed. Sealfon, S. C.) 366-428 (Academic Press, 1995).

28. Manglik, A. \& Kruse, A. C. Structural Basis for G Protein-Coupled Receptor Activation. 
Biochemistry 56, 5628-5634 (2017).

29. Huang, W. et al. Structural insights into $\mu$-opioid receptor activation. Nature 524, 315-321 (2015).

30. Lin, X. et al. Structural basis of ligand recognition and self-activation of orphan GPR52. Nature 579, 152-157 (2020).

31. Kim, K. et al. Structure of a Hallucinogen-Activated Gq-Coupled 5-HT2A Serotonin Receptor. Cell 182, 1574-1588.e19 (2020).

32. Maeda, S., Qu, Q., Robertson, M. J., Skiniotis, G. \& Kobilka, B. K. Structures of the M1 and M2 muscarinic acetylcholine receptor/G-protein complexes. Science 364, 552-557 (2019).

33. Zhang, Y. et al. Cryo-EM structure of the activated GLP-1 receptor in complex with a G protein. Nature 546, 248-253 (2017).

34. García-Nafría, J., Lee, Y., Bai, X., Carpenter, B. \& Tate, C. G. Cryo-EM structure of the adenosine A2A receptor coupled to an engineered heterotrimeric G protein. Elife 7, (2018).

35. Kato, H. E. et al. Conformational transitions of a neurotensin receptor 1-Gi1 complex. Nature 572, 80-85 (2019).

36. Zhang, M. et al. Cryo-EM structure of an activated GPCR--G protein complex in lipid nanodiscs. Nat. Struct. Mol. Biol. 1-10 (2021).

37. Mirzadegan, T., Benkö, G., Filipek, S. \& Palczewski, K. Sequence analyses of G-protein-coupled receptors: similarities to rhodopsin. Biochemistry 42, 2759-2767 (2003).

38. Schöppe, J. et al. Crystal structures of the human neurokinin 1 receptor in complex with clinically used antagonists. Nat. Commun. 10, 17 (2019).

39. Valentin-Hansen, L., Frimurer, T. M., Mokrosinski, J., Holliday, N. D. \& Schwartz, T. W. Biased $\mathrm{Gs}$ versus $\mathrm{Gq}$ proteins and $\beta$-arrestin signaling in the NK1 receptor determined by interactions in the water hydrogen bond network. J. Biol. Chem. 290, 24495-24508 (2015). 
40. White, J. F. et al. Structure of the agonist-bound neurotensin receptor. Nature 490, 508-513 (2012).

41. Koehl, A. et al. Structure of the $\mu$-opioid receptor-Gi protein complex. Nature 558, 547-552 (2018).

42. Hong, C. et al. Structures of active-state orexin receptor 2 rationalize peptide and small-molecule agonist recognition and receptor activation. Nat. Commun. 12, 815 (2021).

43. Yin, J. et al. Crystal structure of the human NK1 tachykinin receptor. Proc. Natl. Acad. Sci. U. S. A. 115, 13264-13269 (2018).

44. Chen, S. et al. Human substance $P$ receptor binding mode of the antagonist drug aprepitant by NMR and crystallography. Nat. Commun. 10, 638 (2019).

45. Fong, T. M. et al. Interaction of glutamine 165 in the fourth transmembrane segment of the human neurokinin-1 receptor with quinuclidine antagonists. J. Biol. Chem. 269, 14957-14961 (1994).

46. Holst, B., Zoffmann, S., Elling, C. E., Hjorth, S. A. \& Schwartz, T. W. Steric Hindrance Mutagenesis versus Alanine Scan in Mapping of Ligand Binding Sites in the Tachykinin NK1 Receptor. Mol. Pharmacol. 53, 166-175 (1998).

47. Fong, T. M. et al. Mutational analysis of neurokinin receptor function. Can. J. Physiol. Pharmacol. 73, 860-865 (1995).

48. Fong, T. M. et al. The role of histidine 265 in antagonist binding to the neurokinin-1 receptor. J. Biol. Chem. 269, 2728-2732 (1994).

49. Huang, R. R. C., Yu, H., Strader, C. D. \& Fong, T. M. Interaction of Substance P with the Second and Seventh Transmembrane Domains of the Neurokinin-1 Receptor. Biochemistry 33, 3007-3013 (1994).

50. Holst, B., Hastrup, H., Raffetseder, U., Martini, L. \& Schwartz, T. W. Two active molecular 
phenotypes of the tachykinin NK1 receptor revealed by G-protein fusions and mutagenesis.

J. Biol. Chem. 276, 19793-19799 (2001).

51. Suomivuori, C.-M. et al. Molecular mechanism of biased signaling in a prototypical G protein-coupled receptor. Science 367, 881-887 (2020).

52. Ma, Y. et al. Structural Basis for Apelin Control of the Human Apelin Receptor. Structure 25, 858-866.e4 (2017).

53. Thompson, G. L. et al. Biased Agonism of Endogenous Opioid Peptides at the $\mu$-Opioid Receptor. Mol. Pharmacol. 88, 335-346 (2015).

54. Gomes, I. et al. Biased signaling by endogenous opioid peptides. Proc. Natl. Acad. Sci. U. S. A. $117,11820-11828$ (2020).

55. Liao, Y. et al. Human Neuropeptide S Receptor Is Activated via a Gaq Protein-biased Signaling Cascade by a Human Neuropeptide S Analog Lacking the C-terminal 10 Residues *. J. Biol. Chem. 291, 7505-7516 (2016).

56. Clark, S. D. et al. Identification of the first biased NPS receptor agonist that retains anxiolytic and memory promoting effects with reduced levels of locomotor stimulation. Neuropharmacology 118, 69-78 (2017).

57. Kaiser, A., Wanka, L., Ziffert, I. \& Beck-Sickinger, A. G. Biased agonists at the human Y1 receptor lead to prolonged membrane residency and extended receptor $\mathrm{G}$ protein interaction. Cell. Mol. Life Sci. 77, 4675-4691 (2020).

58. Manglik, A. et al. Crystal structure of the $\mu$-opioid receptor bound to a morphinan antagonist. Nature 485, 321-326 (2012).

59. Egloff, P. et al. Structure of signaling-competent neurotensin receptor 1 obtained by directed evolution in Escherichia coli. Proc. Natl. Acad. Sci. U. S. A. 111, E655-62 (2014).

60. Dror, R. O. et al. Structural basis for nucleotide exchange in heterotrimeric G proteins. 
Science 348, 1361-1365 (2015).

61. Mastronarde, D. N. Automated electron microscope tomography using robust prediction of specimen movements. J. Struct. Biol. 152, 36-51 (2005).

62. Zheng, S. Q. et al. MotionCor2: anisotropic correction of beam-induced motion for improved cryo-electron microscopy. Nat. Methods 14, 331-332 (2017).

63. Punjani, A., Rubinstein, J. L., Fleet, D. J. \& Brubaker, M. A. cryoSPARC: algorithms for rapid unsupervised cryo-EM structure determination. Nat. Methods 14, 290-296 (2017).

64. Asarnow, D., Palovcak, E. \& Cheng, Y. asarnow/pyem: UCSF pyem v0.5. (2019). doi:10.5281/zenodo.3576630.

65. Scheres, S. H. W. RELION: implementation of a Bayesian approach to cryo-EM structure determination. J. Struct. Biol. 180, 519-530 (2012).

66. Grant, T., Rohou, A. \& Grigorieff, N. cisTEM, user-friendly software for single-particle image processing. eLife vol. 7 (2018).

67. Dang, S. et al. Cryo-EM structures of the TMEM16A calcium-activated chloride channel. Nature 552, 426-429 (2017).

68. Pettersen, E. F. et al. UCSF ChimeraX: Structure visualization for researchers, educators, and developers. Protein Sci. 30, 70-82 (2021).

69. Liebschner, D. et al. Macromolecular structure determination using X-rays, neutrons and electrons: recent developments in Phenix. Acta Crystallogr D Struct Biol 75, 861-877 (2019).

70. Afonine, P. V. et al. Real-space refinement in PHENIX for cryo-EM and crystallography. Acta Crystallogr D Struct Biol 74, 531-544 (2018).

71. Williams, C. J. et al. MolProbity: More and better reference data for improved all-atom structure validation. Protein Sci. 27, 293-315 (2018). 
72. Barad, B. A. et al. EMRinger: side chain-directed model and map validation for 3D cryo-electron microscopy. Nat. Methods 12, 943-946 (2015).

73. Afonine, P. V. et al. New tools for the analysis and validation of cryo-EM maps and atomic models. Acta Crystallogr D Struct Biol 74, 814-840 (2018).

74. Jacobson, M. P. et al. A hierarchical approach to all-atom protein loop prediction. Proteins 55, 351-367 (2004).

75. Jacobson, M. P., Friesner, R. A., Xiang, Z. \& Honig, B. On the role of the crystal environment in determining protein side-chain conformations. J. Mol. Biol. 320, 597-608 (2002).

76. Ghanouni, P. et al. The Effect of $\mathrm{pH}$ on $\beta 2$ Adrenoceptor Function: EVIDENCE FOR PROTONATION-DEPENDENT ACTIVATION*. J. Biol. Chem. 275, 3121-3127 (2000).

77. Ranganathan, A., Dror, R. O. \& Carlsson, J. Insights into the role of Asp79(2.50) in $\beta 2$ adrenergic receptor activation from molecular dynamics simulations. Biochemistry 53, 7283-7296 (2014).

78. Zhang, L. \& Hermans, J. Hydrophilicity of Cavities in Proteins. PROTEINS: Structure, Function, and Genetics 24, 433-438 (1996).

79. Lomize, M. A., Lomize, A. L., Pogozheva, I. D. \& Mosberg, H. I. OPM: orientations of proteins in membranes database. Bioinformatics 22, 623-625 (2006).

80. Betz, R. M. Dabble. (2017). doi:10.5281/zenodo.836914.

81. Beglov, D. \& Roux, B. Finite representation of an infinite bulk system: Solvent boundary potential for computer simulations. J. Chem. Phys. 100, 9050-9063 (1994).

82. Huang, J. et al. CHARMM36m: an improved force field for folded and intrinsically disordered proteins. Nat. Methods 14, 71-73 (2017).

83. Klauda, J. B. et al. Update of the CHARMM all-atom additive force field for lipids: validation 
on six lipid types. J. Phys. Chem. B 114, 7830-7843 (2010).

84. D.A. Case, K. Belfon, I.Y. Ben-Shalom, S.R. Brozell, D.S. Cerutti, T.E. Cheatham, III, V.W.D. Cruzeiro, T.A. Darden, R.E. Duke, G. Giambasu, M.K. Gilson, H. Gohlke, A.W. Goetz, R. Harris, S. Izadi, S.A. Izmailov, K. Kasavajhala, A. Kovalenko, R. Krasny, T. Kurtzman, T.S. Lee, S. LeGrand, P. Li, C. Lin, J. Liu, T. Luchko, R. Luo, V. Man, K.M. Merz, Y. Miao, O. Mikhailovskii, G. Monard, H. Nguyen, A. Onufriev, F.Pan, S. Pantano, R. Qi, D.R. Roe, A. Roitberg, C. Sagui, S. Schott-Verdugo, J. Shen, C. Simmerling, N.R.Skrynnikov, J. Smith, J. Swails, R.C. Walker, J. Wang, L. Wilson, R.M. Wolf, X. Wu, Y. Xiong, Y. Xue, D.M. York and P.A. Kollman. AMBER 2018. University of California, San Francisco (2018).

85. Salomon-Ferrer, R. et al. Routine microsecond molecular dynamics simulations with AMBER on GPUs. 1. Generalized Born. J. Chem. Theory Comput. 8, 1542-1555 (2012).

86. Hopkins, C. W., Le Grand, S., Walker, R. C. \& Roitberg, A. E. Long-Time-Step Molecular Dynamics through Hydrogen Mass Repartitioning. J. Chem. Theory Comput. 11, 1864-1874 (2015).

87. Ryckaert, J. P., Ciccotti, G. \& Berendsen, H. J. C. Numerical integration of the Cartesian Equations of Motion of a System with Constraints: Molecular Dynamics of n-Alkanes. J. Comput. Phys. 23, 321-341 (1977).

88. Roe, D. R. \& Cheatham, T. E., 3rd. PTRAJ and CPPTRAJ: Software for Processing and Analysis of Molecular Dynamics Trajectory Data. J. Chem. Theory Comput. 9, 3084-3095 (2013).

89. Humphrey, W., Dalke, A. \& Schulten, K. VMD: visual molecular dynamics. J. Mol. Graph. 14, 33-8, 27-8 (1996).

90. Michaud-Agrawal, N., Denning, E. J., Woolf, T. B. \& Beckstein, O. MDAnalysis: a toolkit for the analysis of molecular dynamics simulations. J. Comput. Chem. 32, 2319-2327 (2011). 